\title{
REVIEW
}

\section{Pulsar wind nebulae created by fast-moving pulsars}

\author{
O. Kargaltsev ${ }^{1} \dagger$, G. G. Pavlov ${ }^{2}$, N. Klingler ${ }^{1}$ and B. Rangelov ${ }^{1,3}$ \\ ${ }^{1}$ Department of Physics, George Washington University, Washington, DC 20052, USA \\ ${ }^{2}$ Department of Astronomy and Astrophysics, Pennsylvania State University, University Park, \\ PA 16802, USA \\ ${ }^{3}$ Department of Physics, Texas State University, San Marcos, TX 78666, USA
}

(Received 16 April 2017; revised 2 August 2017; accepted 3 August 2017)

\begin{abstract}
We review multiwavelength properties of pulsar wind nebulae created by supersonically moving pulsars and the effects of pulsar motion on the pulsar wind nebulae morphologies and the ambient medium. Supersonic pulsar wind nebulae are characterized by bow-shaped shocks around the pulsar and/or cometary tails filled with the shocked pulsar wind. In the past several years significant advances in supersonic pulsar wind nebula studies have been made in deep observations with the Chandra and XMM-Newton X-ray observatories and the Hubble Space Telescope. In particular, these observations have revealed very diverse supersonic pulsar wind nebula morphologies in the pulsar vicinity, different spectral behaviours of long pulsar tails, the presence of puzzling outflows misaligned with the pulsar velocity and far-UV bow shocks. Here we review the current observational status focusing on recent developments and their implications.
\end{abstract}

Key words: astrophysical plasmas

\section{Introduction}

Pulsars and their winds are among nature's most powerful particle accelerators, producing particles with energies up to a few PeV. In addition to high-energy radiation from the magnetosphere, the rotational (spin) energy of a neutron star is carried away in the form of a magnetized ultra-relativistic pulsar wind (PW), whose non-thermal emission can be seen from radio to $\gamma$-rays, with energies reaching nearly $100 \mathrm{TeV}$ Aharonian et al. (2004). Recent X-ray and $\gamma$-ray observations suggest that the ratio of energy radiated from the 'observable' pulsar wind nebula (PWN) to the energy radiated from the magnetosphere can vary significantly among pulsars. For instance, for the Crab pulsar and its PWN the ratio of the luminosities integrated over the entire electromagnetic spectrum $^{1}$ is $L_{\mathrm{PWN}} / L_{\mathrm{PSR}} \simeq 30$ while for the Vela pulsar and

$†$ Email address for correspondence: kargaltsev@gwu.edu

${ }^{1}$ The estimates of the Crab's PWN and pulsar luminosities are based on the spectra shown in figure 2 of Bühler \& Blandford (2014). The Vela PWN luminosity is estimated from the spectrum shown in figure 4 of Mattana et al. (2011) which corresponds to the $r=6^{\prime}$ region around the pulsar. The Vela pulsar's luminosity is based on figure 28 of Kuiper \& Hermsen (2015). 
its compact PWN it is $\simeq 0.03$. The ratio becomes $\sim 1$ if the luminosity ${ }^{2}$ of Vela $\mathrm{X}$ (a large structure south of the pulsar bright in radio, X-rays, and high-energy $\gamma$-rays; Grondin et al. 2013) is added to the compact PWN luminosity.

The speed of the PW is highly relativistic immediately beyond the pulsar magnetosphere. However, the interaction with the ambient medium causes the wind to slow down abruptly at a termination shock. Immediately downstream of the termination shock, the flow speed is expected to become mildly relativistic, lower than the speed of sound in ultra-relativistic magnetized plasma but much higher than the sound speed in the ambient medium. It is commonly assumed that the distance to the termination shock from the pulsar, $R_{\mathrm{TS}} \sim\left(\dot{E} / 4 \pi c P_{\mathrm{amb}}\right)^{1 / 2}$, can be estimated by balancing the PW pressure ${ }^{3}$ with the ambient pressure. The flow keeps decelerating further downstream and reaches the contact discontinuity that separates the shocked PW from the surrounding medium shocked in the forward shock ${ }^{4}$. For a stationary (or slowly moving, subsonic) pulsar, the contact discontinuity sphere (hence the PWN) is expected to be expanding as the pulsar pumps more energy into it, until the radiative and/or adiabatic expansion losses balance the energy input. The shocked PW within the PWN bubble contains ultra-relativistic particles with randomized pitch angles and a magnetic field whose structure also becomes somewhat disordered (see three-dimensional (3-D) simulations of the Crab PWN; Porth et al. 2016). Therefore, $\mathrm{PWNe}$ are expected to produce synchrotron radiation, responsible for the PWN emission from radio frequencies through $\mathrm{X}$-rays and into the $\mathrm{MeV}$ range, and inverse-Compton radiation in the $\mathrm{GeV}-\mathrm{TeV}$ range (see e.g. the reviews by Kargaltsev \& Pavlov 2008, 2010; Kargaltsev et al. 2015; Reynolds et al. 2017).

In general, studying PWNe provides information about the pulsars that power them, the properties of the surrounding medium, and the physics of the wind-medium interactions. The detailed structure of the interface between the PW and the surrounding ambient medium is not well understood. In an idealized hydrodynamic scenario, the contact discontinuity separates the shocked interstellar medium (ISM) from the shocked PW. Being compressed and heated at the forward shock, the shocked ambient medium emits radiation in spectral lines and continuum. It has been notoriously difficult to identify the forward shock around many young and bright PWNe residing in supernova remnants (SNRs) (including the Crab PWN). However, there is a class of PWNe, associated with supersonically moving pulsars, where both the shocked ambient medium and the shocked PW can be seen. In this review we will focus on observational properties of supersonic pulsar wind nebulae (SPWNe). For a recent theoretical review, see Bykov et al. (2017).

\subsection{SPWNe - PWNe of supersonic pulsars}

In addition to the ISM pressure, the PWN size and morphology can be significantly affected by the ram pressure of the external medium caused by the fast (supersonic) pulsar motion. Indeed, average pulsar 3-D velocities have been found to be $v_{p} \sim 400 \mathrm{~km} \mathrm{~s}^{-1}$ for an isotropic velocity distribution (Hobbs et al. 2005). This implies that the majority of pulsars only stay within their host SNR environment

\footnotetext{
${ }^{2}$ Vela $\mathrm{X}$ is believed to be a relic PWN filled with PW particles which were produced when the pulsar had a higher $\dot{E}$. The Vela $\mathrm{X}$ luminosity is estimated from the spectrum shown in figure 4 of Mattana et al. (2011).

${ }^{3}$ Although this is a commonly used estimate, rigorously speaking, instead of $\dot{E}$ one should use a fraction of $\dot{E}$ which is associated with the wind and can vary from pulsar to pulsar (see the discussion above).

${ }^{4}$ In reality, various instabilities may lead to a more complex picture where the contact discontinuity is distorted and possibly destroyed.
} 
for a few tens of kilo-years, although some particularly fast-moving pulsars can leave it even earlier. Once the pulsar leaves its host SNR, it enters a very different environment, with a much lower sound speed, $c_{s} \sim 3-30 \mathrm{~km} \mathrm{~s}^{-1}$, depending on the ISM phase ${ }^{5}$, hence the pulsar motion becomes highly supersonic, i.e. $v_{p} / c_{s} \equiv \mathcal{M} \gg 1$, where $\mathcal{M}$ is the Mach number.

The supersonic motion strongly modifies the PWN appearance and the properties of its emission, making it useful to introduce a separate category of SPWNe. In particular, for an isotropic PW, the forward shock, contact discontinuity, and termination shock shapes resemble paraboloids in the pulsar vicinity but have quite different shapes behind the pulsar (see figure 9 in Gaensler et al. (2004)). The distance from the pulsar to the apex of the contact discontinuity can be estimated as

$$
R_{a} \approx\left[\frac{\dot{E} f_{\Omega}}{4 \pi c\left(P_{\mathrm{amb}}+P_{\mathrm{ram}}\right)}\right]^{1 / 2}
$$

At this distance, the PW pressure, $P_{w}=\dot{E} f_{\Omega}\left(4 \pi c r^{2}\right)^{-1}\left(f_{\Omega}\right.$ takes into account PW anisotropy), is balanced by the sum of the ambient pressure, $P_{\mathrm{amb}}=\rho k T\left(\mu m_{H}\right)^{-1}=$ $1.38 \times 10^{-12} n_{H} \mu^{-1} T_{4} \mathrm{dyn} \mathrm{cm}^{-2}$, and the ram pressure, $P_{\text {ram }}=\rho v^{2}=1.67 \times$ $10^{-10} n v_{7}^{2}$ dyn $\mathrm{cm}^{-2}\left(T_{4}=T / 10^{4} \mathrm{~K}, v_{7}=v / 10^{7} \mathrm{~cm} \mathrm{~s}^{-1}, \mu\right.$ is the mean molecular weight, and $n=\rho / m_{H}$ is in units of $\mathrm{cm}^{-3}$ ). Assuming $P_{\text {ram }} \gg P_{\text {amb }}($ or $\mathcal{M} \gg 1$ ), we obtain $R_{a}=6.5 \times 10^{16} n^{-1 / 2} f_{\Omega}^{1 / 2} \dot{E}_{36}^{1 / 2} v_{7}^{-1} \mathrm{~cm}$ (see e.g. Kargaltsev \& Pavlov 2007).

The shocked PW, whose synchrotron emission can be seen in X-rays and radio, is confined between the termination shock and contact discontinuity surfaces. For $\mathcal{M} \gg 1$ and a nearly isotropic preshock wind with a small magnetization parameter (see §2.2), the termination shock acquires a bullet-like shape (Gaensler et al. 2004; Bucciantini, Amato \& Del Zanna 2005). The length of the bullet and the diameter of the post-termination shock $\mathrm{PWN}$ are $\simeq(5-6) R_{a}$ and $\simeq 4 R_{a}$, respectively (Bucciantini et al. 2005).

Additional complexity arises due to the fact that the PW is likely not isotropic but concentrated toward the equatorial plane of the rotating pulsar. Evidence of such anisotropy, at least in young pulsars, is seen in high-resolution Chandra images (e.g. Kargaltsev \& Pavlov 2008), and it is supported by theoretical modelling (Komissarov \& Lyubarsky 2004). Therefore the appearance of an SPWN is expected to depend on the angle between the velocity vector and the spin axis of the pulsar. Vigelius et al. (2007) demonstrated this by performing 3-D hydrodynamical simulations and introducing a latitudinal dependence of the wind power (with the functional form expected for the split vacuum dipole solution). In addition, inhomogeneities in the ambient medium (Vigelius et al. 2007) and ISM entrainment (Morlino, Lyutikov \& Vorster 2015) are expected to affect the bow shock shape. Observational confirmation of these expectations comes from X-ray and $H \alpha$ (and more recently also far-UV, see below) images of SPWNe.

\section{Current sample of SPWNe}

Currently, there are approximately 30 pulsars whose X-ray, radio, or $H \alpha$ images either clearly show or strongly suggest effects of supersonic motion (see tables 1 and 2). Most of these SPWNe (or SPWN candidates) have been found in X-rays, primarily through high-resolution imaging with Chandra (see figure 2). In eight

\footnotetext{
${ }^{5}$ The sound speed in a middle-aged SNR can be of the order of a few hundred $\mathrm{km} \mathrm{s}^{-1}$.
} 


\begin{tabular}{|c|c|c|c|c|c|c|c|}
\hline \# & Pulsar & Associated object(s) & $\begin{array}{c}d \\
(\mathrm{kpc})\end{array}$ & $\begin{array}{c}\log \dot{E} \\
\left(\operatorname{erg~s}^{-1}\right)\end{array}$ & $\begin{array}{c}\log \tau \\
\mathrm{yrs}\end{array}$ & $\begin{array}{c}B_{11} \\
\left(10^{11} \mathrm{G}\right)\end{array}$ & $\begin{array}{c}v_{\perp} \\
\left(\mathrm{km} \mathrm{s}^{-1}\right)\end{array}$ \\
\hline 1 & J0537-6910 a & N157B & 49.7 & 38.68 & 3.69 & 9.25 & - \\
\hline 2 & B1951+32 & СТВ 80 & 3 & 36.57 & 5.03 & 4.86 & 460 \\
\hline 3 & J1826-1256 & HESS J1825-137 & $\sim 3.9^{\mathrm{c}}$ & 36.56 & 4.16 & 37 & - \\
\hline 4 & B1706-44 & G343.1-2.3 & 2.6 & 36.53 & 4.24 & 31.2 & $\lesssim 100$ \\
\hline 5 & B1757-24 & G5.27-0.9, Duck PWN & 3.8 & 36.41 & 4.19 & 40.4 & 198 \\
\hline 6 & $\mathrm{~J} 1747-2958$ & Mouse PWN & 5 & 36.40 & 4.41 & 24.9 & $306 \pm 43$ \\
\hline 7 & $\mathrm{~J} 1135-6055$ & - & $\sim 2.8^{\mathrm{c}}$ & 36.32 & 4.36 & 30.5 & $<330$ \\
\hline 8 & $\mathrm{~J} 1437-5959$ & G315.9-0.0, Frying Pan PWN & 8 & 36.15 & 5.06 & 7.37 & $\sim 300$ \\
\hline 9 & J1101-6101 & G290.1-0.8, Lighthouse PWN & $\sim 7^{\mathrm{c}}$ & 36.13 & 5.06 & 7.24 & $\sim 2000$ \\
\hline 10 & $\mathrm{~J} 1509-5850$ & - & 4 & 35.71 & 5.19 & 9.14 & $200-600$ \\
\hline 11 & В0906-49 & - & 1 & 35.69 & 5.05 & 12.9 & $\sim 60$ \\
\hline 12 & $\mathrm{~B} 1853+01^{\mathrm{a}}$ & W44 & 3.3 & 35.63 & 4.31 & 75.5 & $400_{-73}^{+114}$ \\
\hline 13 & B0740-28 & - & 2 & 35.28 & 5.2 & 16.9 & $275^{\mathrm{d}}$ \\
\hline 14 & $\mathrm{~B} 1957+20$ & the Black Widow pulsar & 1.73 & 35.20 & 9.18 & 0.002 & $\sim 220$ \\
\hline 15 & $\mathrm{~J} 0538+2817$ & S147 & $1.39^{\mathrm{b}}$ & 34.69 & 5.79 & 7.33 & $357_{-43}^{+59}$ \\
\hline 16 & $\mathrm{~B} 0355+54$ & Mushroom PWN & $1.04^{\mathrm{b}}$ & 34.66 & 5.75 & 8.39 & $61_{-9}^{+12}$ \\
\hline 17 & $\mathrm{~J} 0633+1746$ & Geminga PWN & $0.25^{\mathrm{b}}$ & 34.51 & 5.53 & 16.3 & $\sim 200$ \\
\hline 18 & $\mathrm{~J} 2030+4415$ & - & $\sim 1^{\mathrm{c}}$ & 34.46 & 5.74 & 12.3 & - \\
\hline 19 & $\mathrm{~J} 1741-2054$ & - & 0.3 & 33.97 & 5.59 & 26.8 & 155 \\
\hline 20 & $\mathrm{~J} 2124-3358$ & - & 0.41 & 33.83 & 9.58 & 0.003 & $75^{\mathrm{d}}$ \\
\hline 21 & $\mathrm{~J} 0357+3205$ & Morla PWN & 0.5 & 33.77 & 5.73 & 24.3 & $\sim 2000$ \\
\hline 22 & $\mathrm{~J} 0437-4715$ & - & $0.156^{\mathrm{b}}$ & 33.74 & 9.2 & 0.006 & $104.7 \pm 0.9$ \\
\hline 23 & $\mathrm{~J} 2055+2539^{\mathrm{a}}$ & - & $\sim 0.6^{\mathrm{c}}$ & 33.69 & 6.09 & 11.6 & $\lesssim 2300$ \\
\hline 24 & B1929+10 & - & $0.36^{\mathrm{b}}$ & 33.59 & 6.49 & 5.18 & $\widetilde{C}_{177_{-5}^{+4}}^{+4}$ \\
\hline 25 & B $2224+65$ & Guitar Nebula & 1.88 & 33.07 & 6.05 & 26 & 1626 \\
\hline 26 & - & $\mathrm{IC} 443^{\mathrm{a}}$ & 1.4 & - & - & - & $\sim 250$ \\
\hline 27 & - & MSH 15-56, G326.3-1.8 & 4 & - & - & - & $100-400$ \\
\hline 28 & - & G327.1-1.1, Snail PWN & 7 & - & - & - & $\sim 500$ \\
\hline
\end{tabular}

TABlE 1. Parameters of pulsars with SPWNe (from the Australia Telescope National Facility (ATNF) pulsar catalogue; Manchester et al. 2005). The pulsars are listed in order of decreasing $\dot{E}$. Distance $d$ is given in units of kpc, spin-down energy loss rate $\dot{E}$, pulsar characteristic age $\tau=P / 2 \dot{P}$, surface magnetic field $B_{11}$, and projected pulsar velocity $v_{\perp}$.

Note: Unless specified otherwise the pulsar distances are inferred from the dispersion measure according to Yao, Manchester \& Wang (2017) or taken from the individual papers (see references in table 2).

${ }^{a}$ The supersonic nature of the PWNe powered by these pulsars has not been firmly established.

${ }^{\mathrm{b}}$ Distances are from parallax measurements.

${ }^{c}$ These are radio-quiet pulsars detected in $\gamma$-rays with particularly uncertain distances.

${ }^{\mathrm{d}}$ Velocities are based on the estimates from Brownsberger \& Romani (2014).

cases the supersonic PWN morphologies can be seen in radio. In two cases (PSRs B0906-49 and J1437-5959) radio images clearly show SPWNe which are not seen in X-ray images. Finally, there are eight rotation-powered pulsars with detected $H \alpha$ bow shocks, of which seven have X-ray PWN detections (see table 2), with two bow shocks also detected in far-UV. Despite the existence of $\mathrm{TeV}$ observations, no SPWN detection in $\mathrm{TeV}$ has been reported yet. With the exception of the 


\begin{tabular}{|c|c|c|c|c|c|c|c|c|c|}
\hline \# & Pulsar & $\begin{array}{c}r_{\mathrm{BS}} \\
\left(10^{16} \mathrm{~cm}\right)\end{array}$ & $\begin{array}{c}i \\
\text { (deg.) }\end{array}$ & $\begin{array}{c}l \\
(\mathrm{pc})\end{array}$ & $\begin{array}{c}\log L_{X} \\
\left(\operatorname{erg~s}^{-1}\right)\end{array}$ & $\log \eta_{X}$ & $H \alpha$ & Rad. & Ref. \\
\hline 1 & J0537-6910 & - & - & 3.7 & $36.21 \pm 0.01$ & -2.47 & $\mathrm{~N}$ & $\mathrm{~N}$ & [1] \\
\hline 2 & B1951+32 & $<12$ & - & 1.2 & $33.02 \pm 0.11$ & -3.55 & $?^{\mathrm{a}}$ & $\mathrm{Y}^{\mathrm{a}}$ & {$[2,3]$} \\
\hline 3 & J1826-1256 & - & - & 5.8 & $33.38 \pm 0.06$ & -3.18 & $?$ & $?$ & {$[52,53]$} \\
\hline 4 & B1706-44 & $\sim 70$ & $?$ & 3 & $32.60 \pm 0.10$ & -3.93 & $\mathrm{~N}$ & $\mathrm{Y}$ & [4] \\
\hline 5 & B1757-24 & $<13$ & - & 0.4 & $33.20 \pm 0.14$ & -3.21 & $?$ & $\mathrm{Y}$ & {$[3,35,36]$} \\
\hline 6 & $\mathrm{~J} 1747-2958$ & $\sim 14$ & $\sim 20^{\circ}$ & 1.1 & $33.83 \pm 0.09$ & -2.57 & $?$ & $\mathrm{Y}$ & [5-8] \\
\hline 7 & $\mathrm{~J} 1135-6055$ & - & - & 1.4 & $32.40 \pm 0.04$ & -3.92 & $?$ & $?$ & {$[53,54]$} \\
\hline 8 & J1437-5959 & - & - & $\sim 20$ & - & - & $?$ & $\mathrm{Y}$ & [27] \\
\hline 9 & J1101-6101 & 34 & - & 3.5 & $32.40 \pm 0.40$ & -3.31 & $?$ & $\mathrm{Y}$ & {$[37-40]$} \\
\hline 10 & J1509-5850 & $<0.6$ & $\sim 90^{\circ}$ & 6.5 & $33.05 \pm 0.04$ & -2.66 & Y & $\mathrm{Y}$ & [9-11] \\
\hline 11 & B0906-49 & - & $\sim 90^{\circ}$ & 3.5 & - & -5.86 & $?$ & $\mathrm{Y}$ & {$[50,51]$} \\
\hline 12 & $\mathrm{~B} 1853+01^{\mathrm{b}}$ & $\sim 33$ & - & 1.3 & $32.20 \pm 0.10$ & -2.58 & $\mathrm{~N}$ & $\mathrm{~N}$ & {$[12,13]$} \\
\hline 13 & B0740-28 & $<6$ & - & - & - & - & Y & $?$ & {$[26]$} \\
\hline 14 & B1957+20 & 1.3 & - & 0.3 & $29.73 \pm 0.40$ & -5.14 & $\mathrm{Y}$ & $?$ & {$[33,34]$} \\
\hline 15 & $\mathrm{~J} 0538+2817$ & $<14$ & $\sim 90^{\circ}$ & 0.02 & $31.30 \pm 0.15$ & -3.39 & $\mathrm{~N}$ & $\mathrm{~N}$ & {$[14,15]$} \\
\hline 16 & $\mathrm{~B} 0355+54$ & 0.5 & $<20^{\circ}$ & 1.5 & $31.20 \pm 0.07$ & -3.46 & $?$ & $\mathrm{~N}$ & {$[16,17]$} \\
\hline 17 & $\mathrm{~J} 0633+1746$ & 4 & $>50^{\circ}$ & 0.35 & $29.35 \pm 0.11$ & -5.53 & $?$ & $?$ & [18-20] \\
\hline 18 & $\mathrm{~J} 2030+4415$ & 3.6 & - & 0.07 & $30.49 \pm 0.18$ & -3.97 & Y & $\mathrm{N}$ & {$[27,28]$} \\
\hline 19 & J1741-2054 & 1.1 & $\sim 75^{\circ}$ & 0.5 & $30.21 \pm 0.02$ & -3.76 & $\mathrm{Y}$ & $?$ & {$[29,30]$} \\
\hline 20 & J2124-3358 & 0.8 & - & 0.04 & $28.98 \pm 0.15$ & -4.85 & $\mathrm{Y}$ & $\mathrm{Y}$ & {$[21,26]$} \\
\hline 21 & $\mathrm{~J} 0357+3205$ & 1.3 & $>70^{\circ}$ & 1.3 & $30.07 \pm 0.20$ & -3.70 & $\mathrm{~N}$ & $\mathrm{~N}$ & {$[31,32]$} \\
\hline 22 & J0437-4715 & 0.28 & $\sim 58^{\circ}$ & - & $\sim 28.6$ & -6.2 & $\mathrm{Y}$ & $?$ & {$[25,26]$} \\
\hline
\end{tabular}

TABLE 2. Continued on next page.

very young and energetic PSR J0537-6910 in the large Magellanic cloud (LMC) ( $\tau=4.9 \mathrm{kyr}, \dot{E}=4.9 \times 10^{38} \mathrm{erg} \mathrm{s}^{-1}$ ), which may turn out to be not an SPWN despite the suggestive X-ray PWN morphology ${ }^{6}$, the rest of SPWNe are powered by non-recycled pulsars with ages between $10 \mathrm{kyr}$ and $3 \mathrm{Myr}$, or by much older recycled pulsars; their spin-down powers span the range of $10^{33}-10^{37} \mathrm{erg} \mathrm{s}^{-1}$ (see figure 1).

\section{Magnetic fields and related parameters}

PWN magnetic fields can be estimated from the synchrotron luminosity and the spectral slope measured in a chosen emitting region. For a power-law spectrum with a photon index $\Gamma$, the magnetic field is given by the equation ${ }^{7}$

$$
B=\left[\frac{L\left(v_{m}, v_{M}\right) \sigma_{s}}{\mathcal{A} V} \frac{\Gamma-2}{\Gamma-1.5} \frac{v_{1}^{1.5-\Gamma}-v_{2}^{1.5-\Gamma}}{v_{m}^{2-\Gamma}-v_{M}^{2-\Gamma}}\right]^{2 / 7} .
$$

Here $L\left(v_{m}, v_{M}\right)$ is the synchrotron luminosity measured in the frequency range $v_{m}<v<v_{M}$ from a radiating volume $V, \sigma_{s}=w_{B} / w_{e}$ is the ratio of the energy density

\footnotetext{
${ }^{6}$ The remote LMC location of the pulsar makes it difficult to reliably determine the pulsar velocity (from the PWN morphology or proper motion) and the properties of the ambient medium.

${ }^{7}$ This equation turns into equation (7.14) in Pacholczyk (1970) at $v_{m}=v_{1}, v_{M}=v_{2}$, and $\sigma_{s}=3 / 4$, which minimizes the total energy density $w_{B}+w_{e}$. Such equations are applicable at $\Gamma>2 / 3$, the minimum value of the photon index for optically thin synchrotron radiation for any electron spectral energy distribution (Ginzburg \& Syrovatskii 1964).
} 


$\begin{array}{cccccccccc}23 & \mathrm{~J}^{2055+2539^{\mathrm{b}}} & <1.4 & - & \sim 1.7 & 30.17 \pm 0.03 & -3.53 & ? & ? & {[49]} \\ 24 & \mathrm{~B} 1929+10 & 1 & \sim 60^{\circ} & 1.5 & 29.50 \pm 0.25 & -4.09 & ? & \mathrm{Y} & {[22,23]} \\ 25 & \text { B2224+65 } & \sim 0 & - & 0.6 & 30.18 \pm 0.10 & -2.89 & \mathrm{Y} & \mathrm{N} & {[24]} \\ 26 & \text { IC443 } & \sim 15 & - & 0.65 & 32.82 \pm 0.03 & - & \mathrm{N} & \mathrm{Y} & {[41-43]} \\ 27 & \text { MSH 15-56 } & 29 & - & 3.5 & 32.8 \pm 0.2 & - & ? & \mathrm{Y} & {[44,45]} \\ 28 & \text { G327.1-1.1 } & 99 & - & 5.6 & 33.09 \pm 0.10 & - & ? & \mathrm{Y} & {[46-48]}\end{array}$

TABLE 2 (cntd). Estimated parameters of SPWNe: bow shock apex stand-off distance $r_{\mathrm{BS}}$, the inclination angle $i$ between the pulsar spin axis and our line of sight, the tail length $l$, the X-ray tail luminosity $L_{X}$ (in the $0.5-8 \mathrm{keV}$ band), and the corresponding X-ray efficiency $\eta_{X}=L_{X} / \dot{E}$. References: [1] - Wang et al. (2001), [2] - Moon et al. (2004), [3] - Zeiger et al. (2008), [4] - Romani et al. (2005), [5] - Gaensler et al. (2004), [6] Hales et al. (2009), [7] - Yusef-Zadeh \& Gaensler (2005), [8] - Klingler et al. (in prep), [9] - Ng et al. (2010), [10] - Kargaltsev et al. (2008), [11] - Klingler et al. (2016a), [12] - Petre, Kuntz \& Shelton (2002), [13] - Frail et al. (1996), [14] - Chatterjee et al. (2009), [15] - Ng et al. (2007), [16] - McGowan et al. (2006), [17] - Klingler et al. (2016b), [18] - Caraveo et al. (2003), [19] - Pavlov, Bhattacharyya \& Zavlin (2010), [20] - Posselt et al. (2017), [21] - Hui \& Becker (2006), [22] - Becker et al. (2006), [23] - Misanovic, Pavlov \& Garmire (2008), [24] - Hui et al. (2012), [25] - Deller et al. (2008), [26] Brownsberger \& Romani (2014), [27] - Camilo et al. (2009), [28] - Marelli et al. (2015), [29] - Romani et al. (2010), [30] - Auchettl et al. (2015), [31] - De Luca et al. (2013), [32] - De Luca et al. (2011), [33] - Stappers et al. (2003), [34] - Huang et al. (2012), [35] - Kaspi et al. (2001), [36] - Blazek et al. (2006), [37] - Halpern et al. (2014), [38] - Tomsick et al. (2012), [39] - Pavan et al. (2014), [40] - Pavan et al. (2016), [41] Gaensler et al. (2006), [42] - Acciari et al. (2009), [43] - Swartz et al. (2015), [44] Plucinsky et al. (2002), [45] - Temim et al. (2013), [46] - Slane et al. (2004), [47] Acero (2011), [48] - Ma et al. (2016), [49] - Marelli et al. (2016a), [50] - Gaensler et al. (1998), [51] - Kramer \& Johnston (2008), [52] - Voisin et al. (2016), [53] - this work, [54] - Manchester et al. (2005).

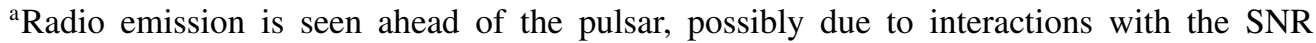
shell; no radio tail is seen. The SNR which contains the PWN is seen in $H_{\alpha}$, making it challenging to discern whether the $H_{\alpha}$ emission is produced by SNR reverse shocks or the PW [2].

${ }^{\mathrm{b}}$ The supersonic nature of the PWNe powered by these pulsars has not been firmly established.

of magnetic field to that of electrons, $\mathcal{A}=\left(e^{2} / 3 m_{e} c^{2}\right)^{2}\left(2 \pi e m_{e} c\right)^{-1 / 2}=3.06 \times 10^{-14}$ in c.g.s. units, and $v_{1}$ and $v_{2}$ are the characteristic synchrotron frequencies $\left(v_{\text {syn }} \simeq\right.$ $\left.e B \gamma^{2} / 2 \pi m_{e} c\right)$ which correspond to the boundary energies $\left(\gamma_{1} m_{e} c^{2}\right.$ and $\left.\gamma_{2} m_{e} c^{2}\right)$ of the electron spectrum $\left(d N_{e} / d \gamma \propto \gamma^{-p} \propto \gamma^{-2 \Gamma+1} ; \gamma_{1}<\gamma<\gamma_{2}\right)$. The magnetic fields in SPWNe estimated from synchrotron brightness and spectral slope measurements with the aid of (3.1) are in the range of $\sim(5-200) \sigma_{s}^{2 / 7} \mu \mathrm{G}$ (see Auchettl et al. 2015; Klingler et al. 2016a,b; Pavan et al. 2016). An additional uncertainty in these estimates is caused, in many cases, by unknown values of the boundary frequencies $v_{1}$ and $v_{2}$, between which the spectrum can be described by a power law. For $\Gamma>1.5$ (as observed in most of the tails), the estimate of $B$ is not sensitive to the $v_{2}$ value as long as $\left(v_{1} / v_{2}\right)^{\Gamma-1.5} \ll 1$. Varying $v_{1}$ from radio $(\sim 1 \mathrm{GHz})$ to $\mathrm{X}$-ray frequencies changes $B$ by a factor of $2-3$, for $1.5<\Gamma<2$. 
(a)

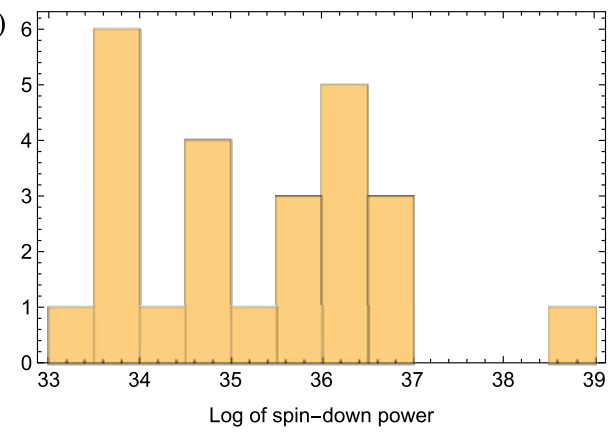

(b)

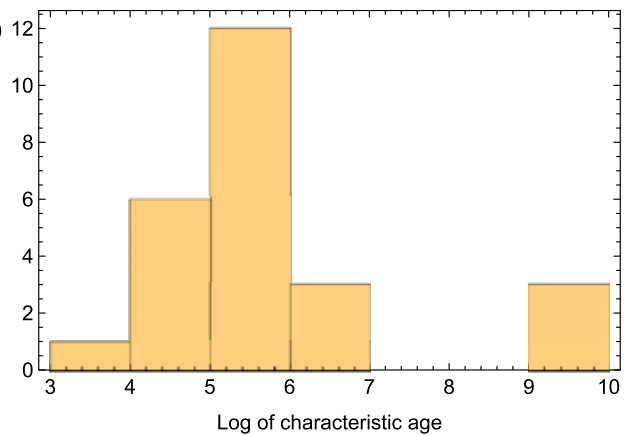

FIGURE 1. $\dot{E}$ ( $a$; in units of $\operatorname{erg~s}^{-1}$ ) and age ( $b$; in years) distributions for the pulsars producing the SPWNe from table 1.

The magnetic field strengths can be used to estimate the Lorentz factors $\gamma$, gyration radii $r_{g}$, and characteristic cooling times $t_{\text {syn }}$ of electrons radiating photons of energy $E$ :

$$
\begin{aligned}
\gamma & \sim 10^{8}(E / 1 \mathrm{keV})^{1 / 2}(B / 20 \mu \mathrm{G})^{-1 / 2}, \\
r_{g} & \sim 10^{14}(E / 1 \mathrm{keV})^{1 / 2}(B / 20 \mu \mathrm{G})^{-3 / 2} \mathrm{~cm} \\
t_{\mathrm{syn}} & \sim 400(E / 1 \mathrm{keV})^{-1 / 2}(B / 20 \mu \mathrm{G})^{-3 / 2} \mathrm{yr} .
\end{aligned}
$$

\section{SPWN heads: connection to viewing angle and pulsar magnetosphere geometry}

As the initially strongly magnetized wind flows away from the pulsar's magnetosphere, its magnetic energy is likely to be at least partly converted into the particle energy. According to the currently-popular models, this occurs due to magnetic field reconnection in a region around the equatorial plane. If the pulsar is an oblique rotator (i.e. its magnetic dipole axis is inclined to the spin axis), one expects 'corrugated' current sheets to be formed, with regions of oppositely directed magnetic fields susceptible to reconnection (see e.g. Lyubarsky \& Kirk 2001 for details). Since the size of the reconnection region is expected to be larger for larger obliquity angles $\alpha$, the magnetic-to-kinetic energy conversion may be more efficient for pulsars with larger $\alpha$. Such pulsars may exhibit brighter PWNe, with more pronounced equatorial components (e.g. the Crab PWN). It is less clear what happens at small $\alpha$; likely, the magnetic-to-kinetic energy conversion would still take place, but outflows along the spin axis ('jets') would be more pronounced than the equatorial 'tori' ${ }^{8}$ (Bühler \& Giomi 2016). Examples of such morphologies for young, subsonic pulsars are the G11.2-0.3, Kes 75 and MSH 15-52 PWNe, which exhibit relatively less luminous tori in the X-ray images (see figure 3). For SPWNe, the identification of the equatorial and polar components is more challenging.

In the X-ray images shown in figure 2 one can often (but not always; see examples below) identify relatively bright and compact PWN 'heads' accompanied by much dimmer (in terms of surface brightness) extended tails. Recent deep, high-resolution Chandra observations revealed fine structures of several bright heads with contrasting

\footnotetext{
${ }^{8}$ The coexistence of a strong polar outflow with a comparatively weak equatorial one can be reproduced in the Komissarov \& Lyubarsky (2004) model, in which the jet is formed by backflow of the equatorial wind diverted by the magnetic field hoop stress toward the spin axis.
} 


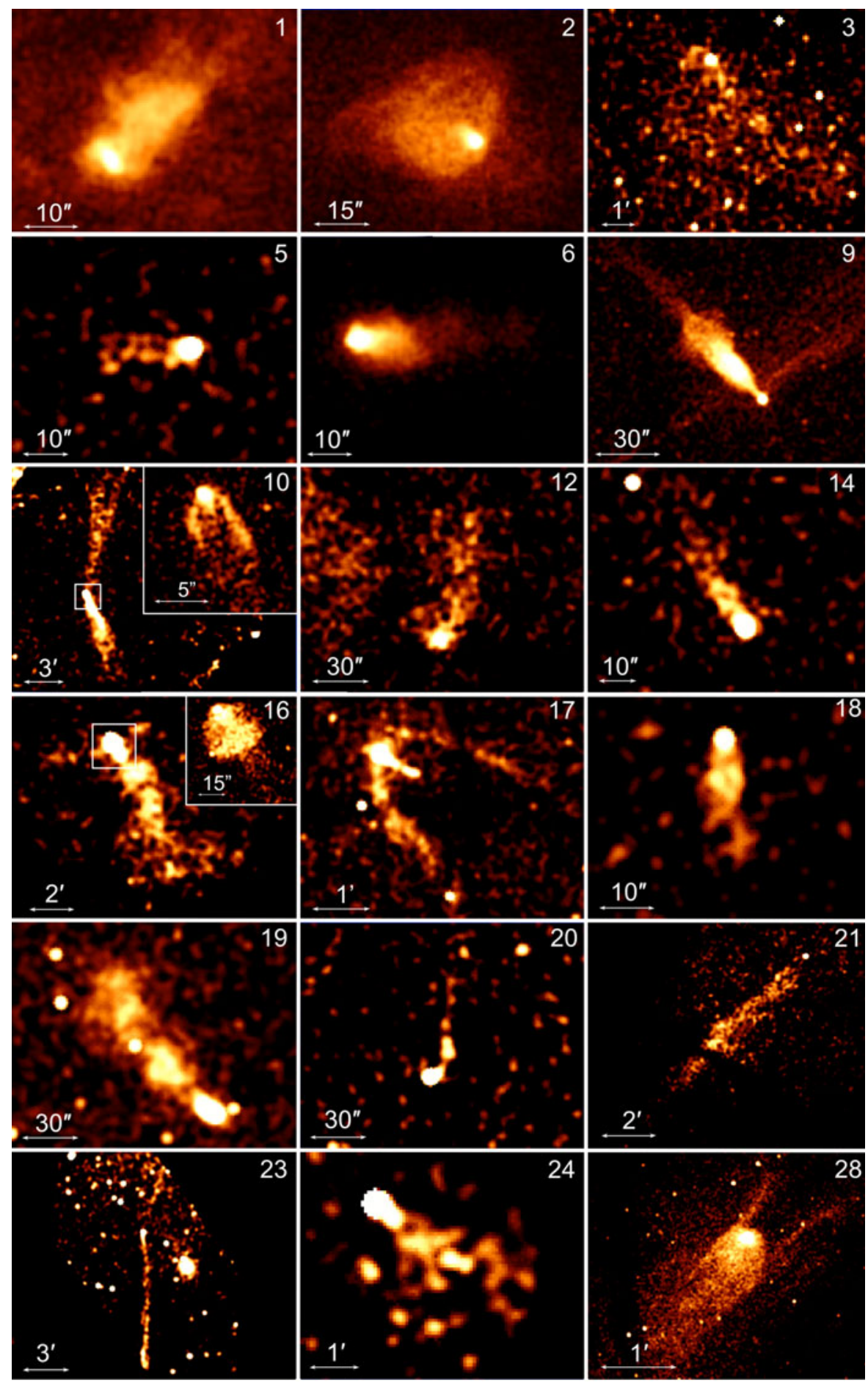

FIgURE 2. Chandra ACIS images of 18 SPWNe. The panels are numbered in accordance with tables 1 and 2. Chandra images of some of these objects are also shown in Reynolds et al. (2017). 

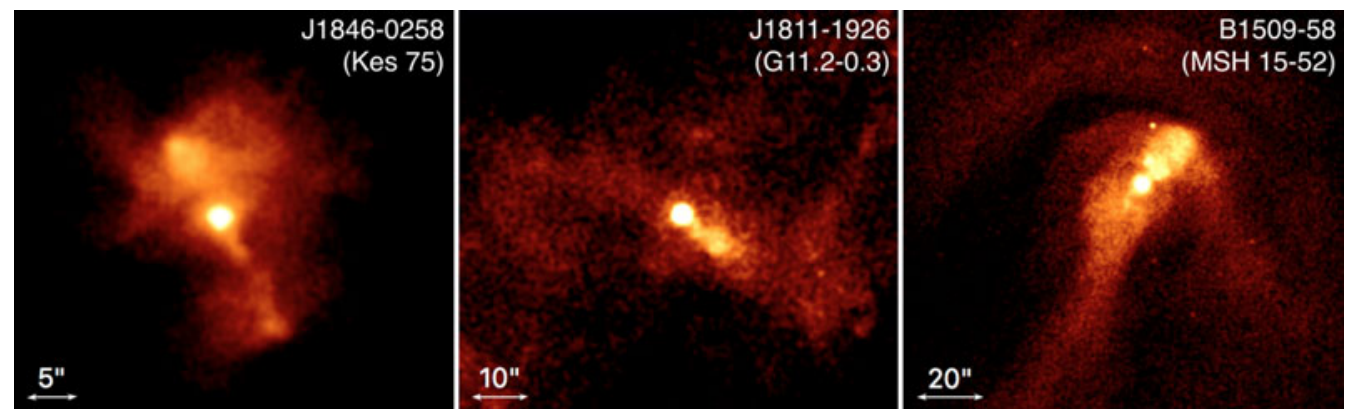

FIGURE 3. Chandra ACIS images of PWNe where axial outflows (along the pulsar spin axis) dominate equatorial components (tori).

morphologies (cf. the insets in panels 10 and 16 in figure 2). For instance, the head of the B0355+54 PWN looks like a symmetric, filled 'dome', brighter near the axis than on the sides (Klingler et al. 2016b). In contrast, the head of the J1509-5058 PWN, looks like two bent tails (Klingler et al. 2016a), with almost no emission between them, except for a short southwest extension just behind the pulsar ${ }^{9}$. This structure is remarkably similar to the Geminga PWN (Posselt et al. 2017; see panel 17 in figure 2), just the angular size of the latter is larger, in accordance with the smaller distance ( $250 \mathrm{pc}$ versus $4 \mathrm{kpc}$ ). The bow-shaped X-ray emission can be associated with either a limb-brightened shell formed by the shocked PW downstream of the termination shock or pulsar jets bent by the ram pressure of the oncoming ISM. In the former case, a lack of diffuse emission in between the lateral tails would require a non-uniform magnetic field in the emitting shell, possibly caused by amplification of the ISM magnetic field component perpendicular to the pulsar's velocity vector (Posselt et al. 2017). In the latter scenario, the winds of J1509-5058 and Geminga must be dominated by luminous polar components (as in the PWNe shown in figure 3) rather than by the equatorial component (as in the Crab and Vela PWNe). If the lateral tails of the J1509-5850 head are indeed bent jets, it may be difficult to explain the ordered helical magnetic field morphology in the extended tail (as suggested by radio polarimetry; $\mathrm{Ng}$ et al. 2010); such a structure would be more natural for the axially symmetric case (Romanova, Chulsky \& Lovelace 2005), when the pulsar spin axis (hence the jet directions) is co-aligned with the velocity vector.

The quite different 'filled' morphology of the B0355+53 PWN head could be due to different mutual orientations of the pulsar's spin axis, magnetic dipole axis, velocity vector and the line of sight. Some information on these orientations could be inferred from the pulsar's signatures at different energies. For instance, Geminga is a $\gamma$-ray pulsar (as well as PSR B1509-58) with no bright radio emission, while PSR $\mathrm{B} 0355+64$ was not detected in $\gamma$-rays but is quite bright in radio. One can speculate that Geminga and J1509-5850 are moving in the plane of the sky, and their spin axes are nearly perpendicular to the velocity vectors and to the line of sight (this assumption would be consistent with the jet interpretation of the lateral tails). On the contrary, the spin axis of B0355+54 could be nearly aligned with the line of sight, in which case the 'dome' would be interpreted as the equatorial torus distorted by the ram pressure while the central brightening would be the sky projection of the bent

\footnotetext{
${ }^{9}$ Interestingly, the tails look slightly separated from the pulsar similar to the base of the south-eastern inner jet in the Vela PWN; see figure 1 in Levenfish et al. (2013).
} 
jets. Thus, it is quite plausible that the qualitative morphological differences in the appearances of PWN heads can be attributed to geometrical factors (i.e. the angles between the line of sight, velocity vector, spin axis and magnetic dipole axis).

Since the pulsar light curves in different energy ranges should also depend on the same geometrical factors, it is interesting to look for correlations between the PWN head shapes and light curves. For instance, both the $\gamma$-ray and radio light curves of PSRs J1509-5850 and B1706-44 are remarkably similar, not only in shapes but also in phase shifts between the $\gamma$-ray and radio pulses (see figure 4), which implies similar geometries and allows one to expect similar PWN morphologies. We see in figure 4 that the B1706-44 X-ray PWN shows clear jets (without obvious bending in the pulsar vicinity) and a relatively underluminous equatorial component (in contrast to the Crab). Although faint, the large-scale morphology of the B1706-44 PWN suggests that the pulsar is moving at the position angle (east of north) of approximately $80^{\circ}$ ( $\mathrm{Ng} \&$ Romani 2008). Thus, although the PWN head morphologies do not look exactly the same, one could imagine that if B1706-44 were moving faster and had even more pronounced jets, its PWN in the pulsar vicinity could look like the one around J1509-5850. The radio and $\gamma$-ray light curves of the Mouse pulsar (J1747-2958) are similar to those of PSRs J1509-5058 and J1709-4429 (see figure 4). The only difference is that the $\gamma$-ray pulse of PSR J1747-2958 is slightly wider (with a deeper trough) and more asymmetric compared to those of PSRs J1509-5058 and J1709-4429. All three pulsars display single radio peaks with very similar phase separation from the $\gamma$ pulses. According to the outer gap magnetospheric emission models, this implies a fairly large magnetic inclination angle (so that both $\gamma$-ray and radio pulsations can be seen). It is likely that these angles are somewhat larger for PSR J1747-2958 than those of PSRs J1509-5058 and J1709-4429. These considerations help to interpret the appearances of the compact nebulae by suggesting that the equatorial outflow dominates over the polar outflow components in these cases.

Interestingly, we do not see bright heads in the X-ray PWNe of some supersonic pulsars, even those with relatively bright X-ray tails (e.g. PSR J1101-6101 and $\mathrm{J} 0357+3205$; panels 9 and 21 in figure 2). In some of those cases, however, the heads may be too compact to resolve because of high pulsar velocities and large distances.

SPWNe can also display contrasting radio morphologies. X-ray bright PWN heads may or may not be bright in radio. For instance the Mouse PWN has the head which is bright in both X-rays and radio, while there is very little (if any) radio emission from the head of the J1509-5058 PWN, although it is bright in X-rays (see figure 5).

Understanding the causes for the differing head (and, possibly, tail; see §5) morphologies in SPWNe is important because it can help to determine the orientation of the pulsar spin axis with respect to the line of sight and to the pulsar velocity vector (see figure 6). The former is the angle $\zeta$, an important parameter for comparing magnetospheric emission models with the $\gamma$-ray and radio light curves (see e.g. Pierbattista et al. 2016). The angle between the spin axis and the pulsar velocity (the direction of the 'natal' kick for not too old pulsars) has important implications for the supernova explosion models (Ng \& Romani 2007). SPWNe are particularly suitable for this purpose because the projected neutron star velocity can be inferred from the PWN morphology in the absence of neutron star proper motion measurement.

\section{Pulsar tails}

As supersonic pulsars move through the ISM, the ram pressure confines and channels the PW in the direction opposite to the pulsar's relative velocity with respect 


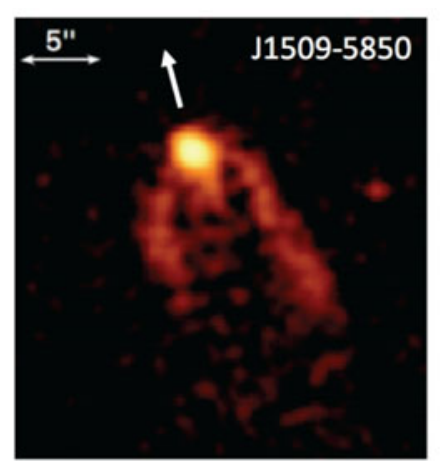

Fermi LAT (black) and radio (red) lightcurves
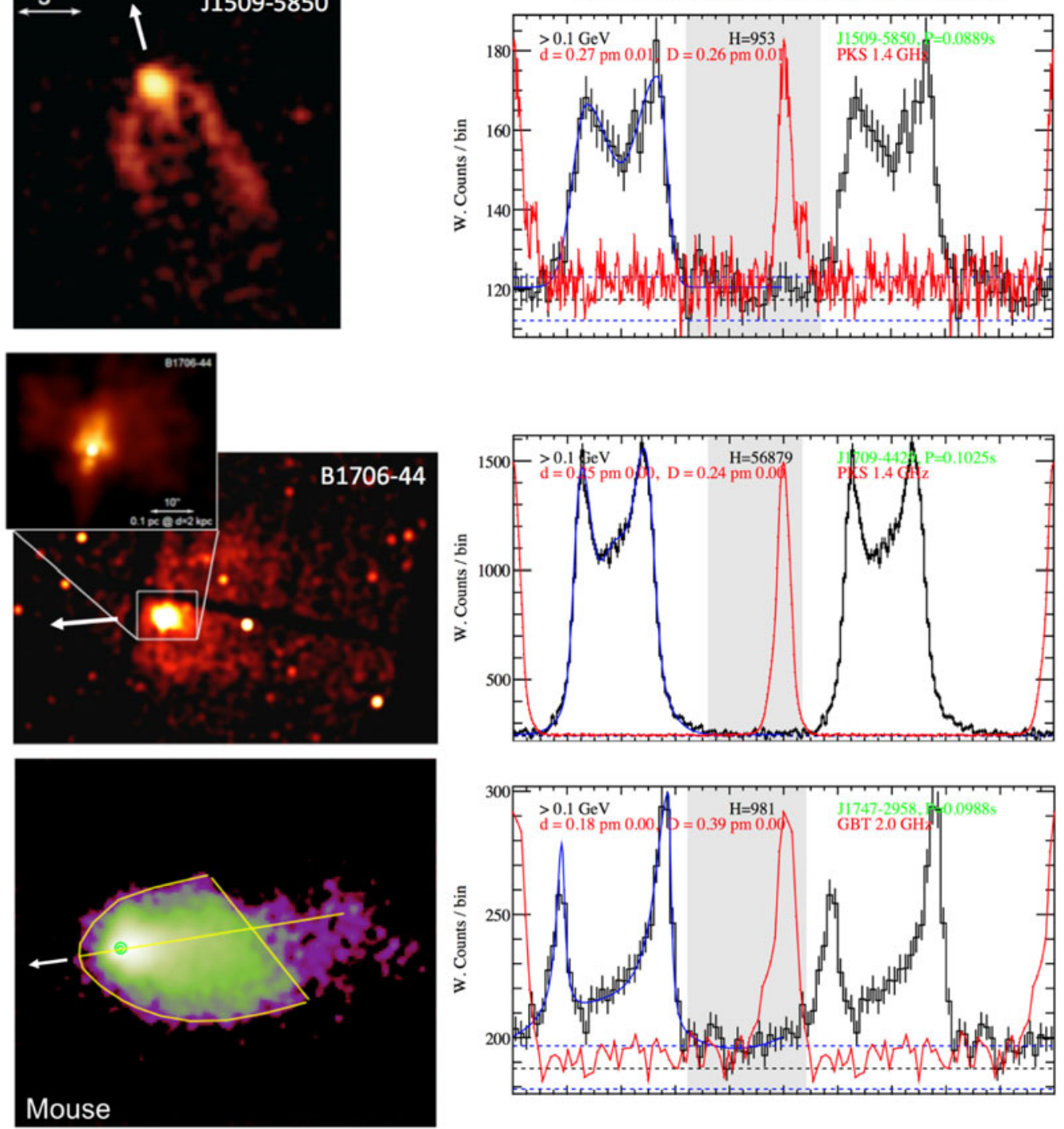

FIGURE 4. Comparison of the PWN morphologies and pulsar light curves for PSRs J1509-5850, B1706-44, and J1747-2858 (top to bottom). The very similar light curves of all three pulsars (both in radio and $\gamma$-rays) suggest similar angles between the spin axis and the line of sight, $\zeta$, and between the spin and magnetic axes, $\alpha$. The contour drawn on top of the Mouse PWN image represents a possible extent of the equatorial outflow affected by the ram pressure (the outflow is in the plane of the shown contour which is symmetric with respect the pulsar velocity direction but appears to be asymmetric once projected onto the sky; Klingler et al. in prep.).

to the local ISM. Therefore, on large spatial scales (compared to the termination shock bullet size) one expects to see a pulsar tail - an extended, ram-pressure-confined structure behind the pulsar (see figures 5 and 7). Several pulsar tails have now been discerned above the background for up to a few parsecs from their parent pulsars. 


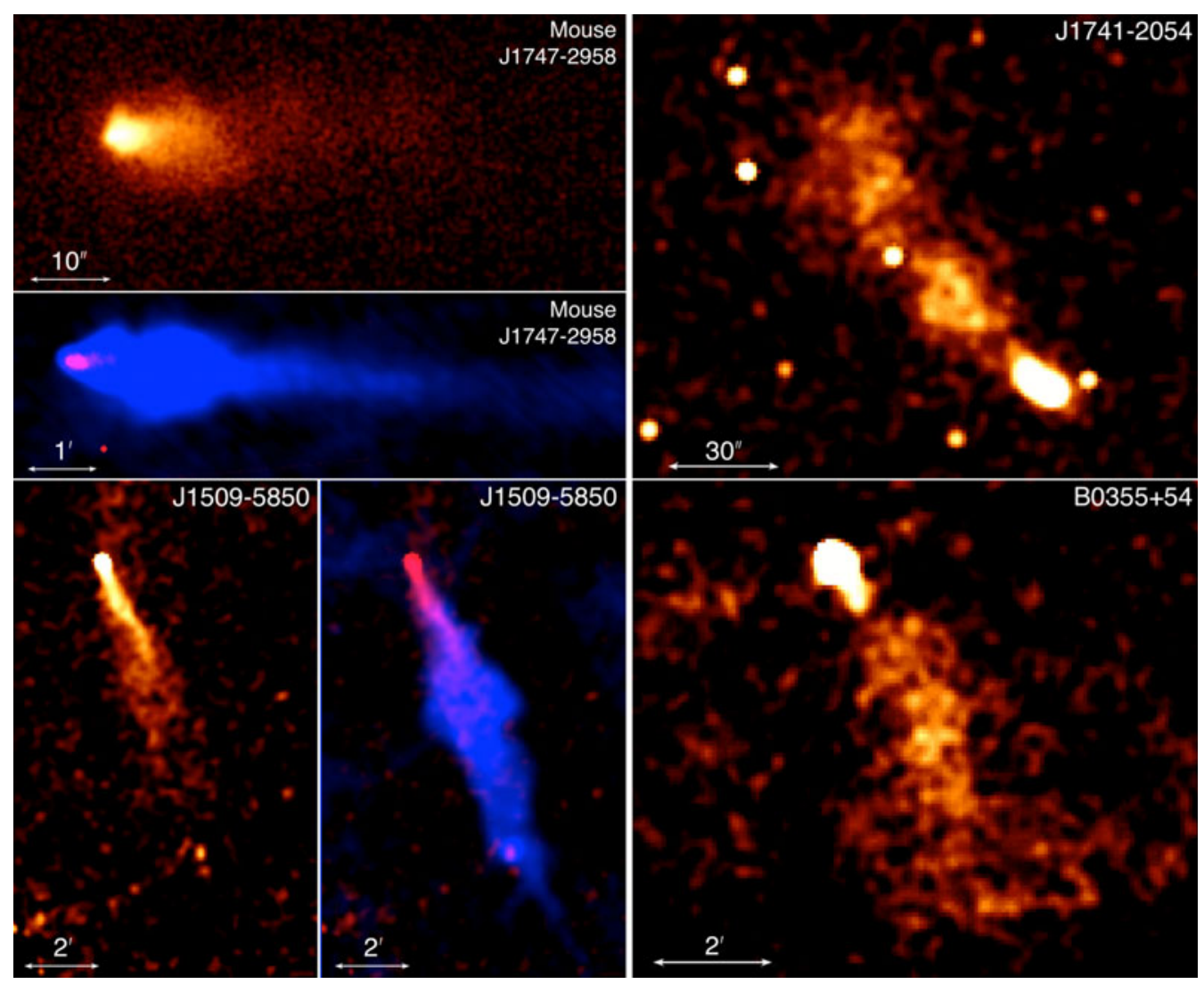

FIGURE 5. Extended tails behind four pulsars. The X-ray images are obtained with Chandra ACIS. For the Mouse PWN and the J1509-5850 PWN, combined X-ray (red) and radio (blue) images are shown.

The longest known tails are those of PSR J1509-5850, whose projected visible length spans $7 \mathrm{pc}$ (at $d=3.8 \mathrm{kpc}$ ) in X-rays and $\sim 10 \mathrm{pc}$ in radio ( $\mathrm{Ng}$ et al. 2010; Klingler et al. 2016a), and the Mouse PWN, whose X-ray and radio tails span 1 and $17 \mathrm{pc}$, respectively (at $d=5 \mathrm{kpc}$; Gaensler et al. 2004).

On large scales, the shapes of many pulsar tails (e.g. B1929+10 - Wang, Li \& Begelman 1993, Becker et al. 2006, Misanovic et al. 2008; J1509-5850 - Klingler et al. 2016a; J1437-5959 - Ng et al. 2012) can be crudely approximated by cones that widen with distance from the pulsar as the outflow slows down and expands (see figure 2). However, in some cases the tail brightness can be strongly non-uniform showing more complex structures that can be described as expanding 'bubbles' (e.g. the SPWNe of PSR J1741-2054; see panel 19 in figure 2; also Auchettl et al. 2015. The bubbles and the rapid widening of tails can be attributed to non-uniformities in the ISM, instabilities in the backflow from the pulsar bow shock (van Kerkwijk \& Ingle 2008), or entrainment (mass loading of the ISM into the PW; Morlino et al. 2015). Some tails, such as those of PSRs J1741-2054 (Auchettl et al. 2015) and B0355+54 (Klingler et al. 2016b), exhibit noticeable 'bendings' at large distances from the pulsars, which could be attributed to ISM winds. Although Chandra, with its superior angular resolution and low background, delivers better images than 

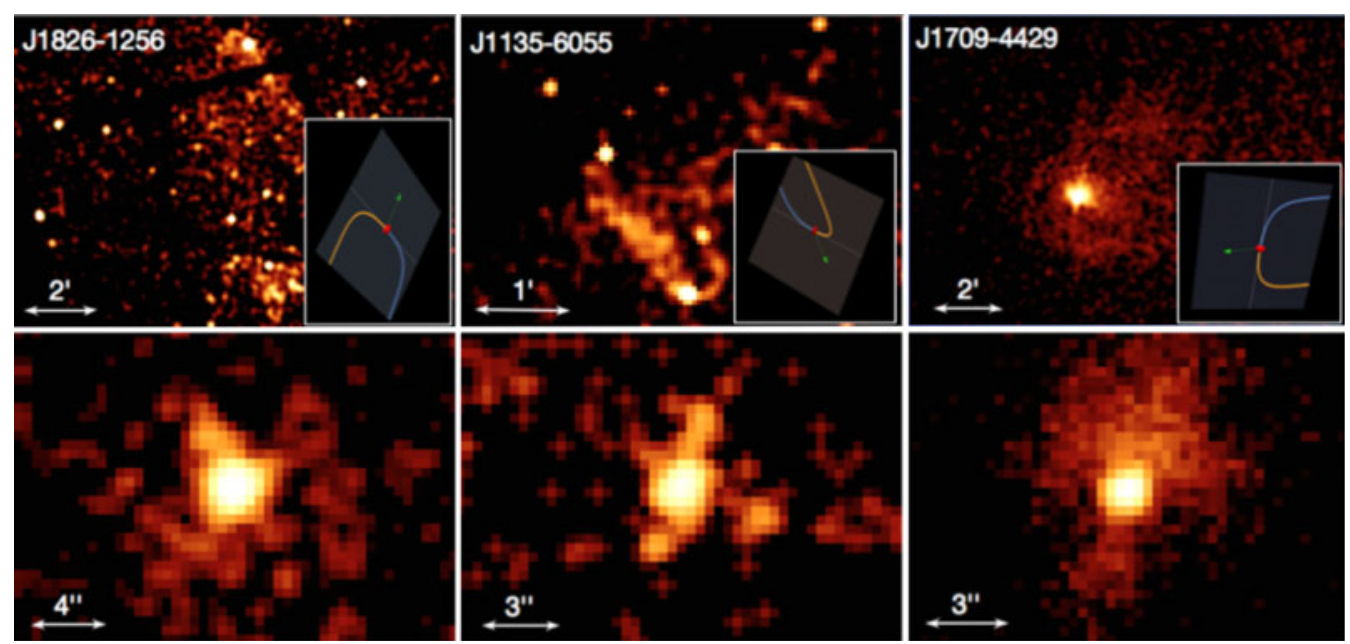

FIGURE 6. Chandra images of SPWNe that likely move with mildly supersonic velocities. A schematic diagram of a possible geometry is shown for each object, with the jets bent by the ram pressure. The green arrows indicate the inferred direction the velocity vector.

XMM-Newton for most pulsar tails, some tails have been studied with XMM-Newton as well (e.g. B1929+10 - Becker et al. 2006; J2055+2539 - Marelli et al. 2016a).

Radio polarimetry of two extended tails (the Mouse and J1509-5058; Yusef-Zadeh \& Gaensler 2005 and $\mathrm{Ng}$ et al. 2010) shows that the magnetic field direction is predominantly transverse in the J1509-5058 tail while it is aligned with the tail in the case of the Mouse. This could indicate that the spin axis is more aligned with the velocity vector in J1509-5058 than in J1747-2858 (see figure 3 in Romanova et al. 2005). This, however, would be at odds with the jet interpretation of the lateral outflows in the J1509-5058 PWN head.

Furthermore, the brightness of the radio tail in the Mouse decreases with distance from the pulsar, whereas in the J1509-5850 and J1101-6101 tails the radio brightness increases with the distance from the pulsar and peaks around $4 \mathrm{pc}$ and $1.7 \mathrm{pc}$ (at $d=3.8 \mathrm{kpc}$ for $\mathrm{J} 1509-5850$ and $d=7 \mathrm{kpc}$ for $\mathrm{J} 1101-6101)$. Such radio surface brightness behaviour could be explained if the PWN magnetic field becomes stronger further down the tail. It is possible for a helical magnetic field configuration if the flow velocity, $v_{\text {tail }}$, decreases rapidly enough with distance from the pulsar, which may lead to an increase in the magnetic field strength $\left(B_{\text {tail }} \propto v_{\text {tail }}^{-1} S_{\text {tail }}^{-1 / 2}\right.$, where $S_{\text {tail }}$ is the crosssectional area of the tail; see e.g. Bucciantini et al. 2005). The G327.1-1.1 (Snail) PWNe contains an undetected pulsar whose tail radio brightness remains constant over its 1'.5 length until it connects to a spherical structure (most likely a reverse shock, as the PWN is located inside its SNR; see figure 7; Ma et al. 2016).

The PWN of PSR J1437-5959 (the Frying Pan; figure 7) is not seen in X-rays, although it is prominent in radio and displays a long radio tail which fades with distance from the pulsar until it becomes brighter near the shell of the alleged SNR G315.9-0.0 (see Ng et al. 2012). Another possible example of such a PWN is the one of PSR B0906-49 (J0908-4913) which has been discovered in radio (Gaensler et al. 1998) but was not detected in the subsequent Chandra ACIS observation (Kargaltsev et al. 2012). It is currently unclear what makes these PWNe so underluminous in X-rays. A natural explanation could be a lack of sufficiently energetic particles which 

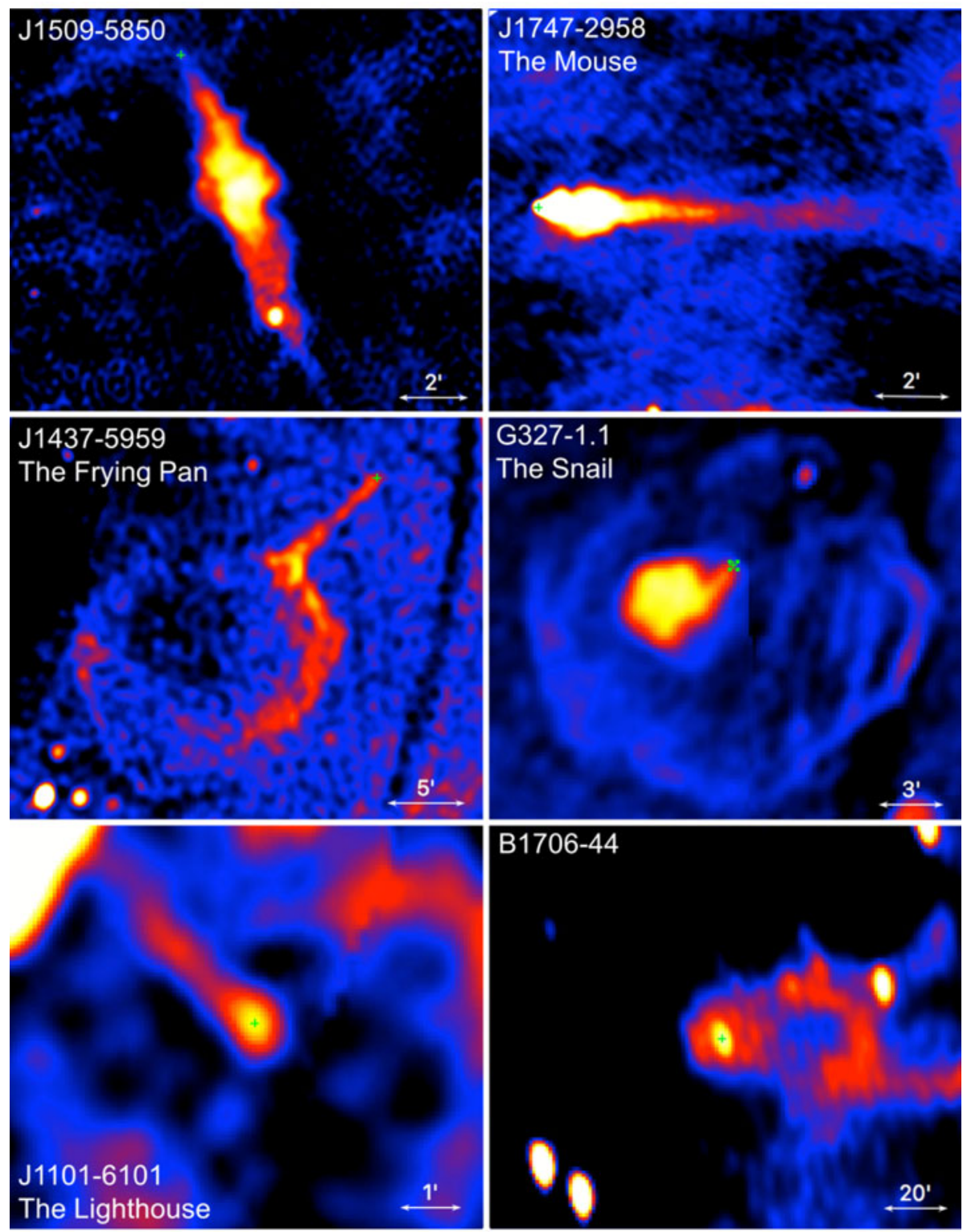

FIGURE 7. Radio images of pulsar tails: J1509-5850 (ATCA, 5 GHz), J1747-2958 (VLA, $1.5 \mathrm{GHz}$ ), J1437-5959 (MOST, 843 MHz), G327-1.1 (MOST, 843 MHz), J1101-6101 (MOST, $843 \mathrm{MHz}$ ), and B1706-44 (VLA, $1.4 \mathrm{GHz}$ ). The green crosses mark the positions of the pulsars (for the Snail no pulsations are detected and the cross shows the position of the X-ray point source).

might be due to a magnetosphere geometry unfavourable for accelerating particles to high energies. However, for PSR B0906-49, radio timing observations suggest that it is an orthogonal rotator with the pulsar's spin axis and direction of motion aligned (Kramer \& Johnston 2008), thus making it similar to some pulsars with bright X-ray PWNe. 

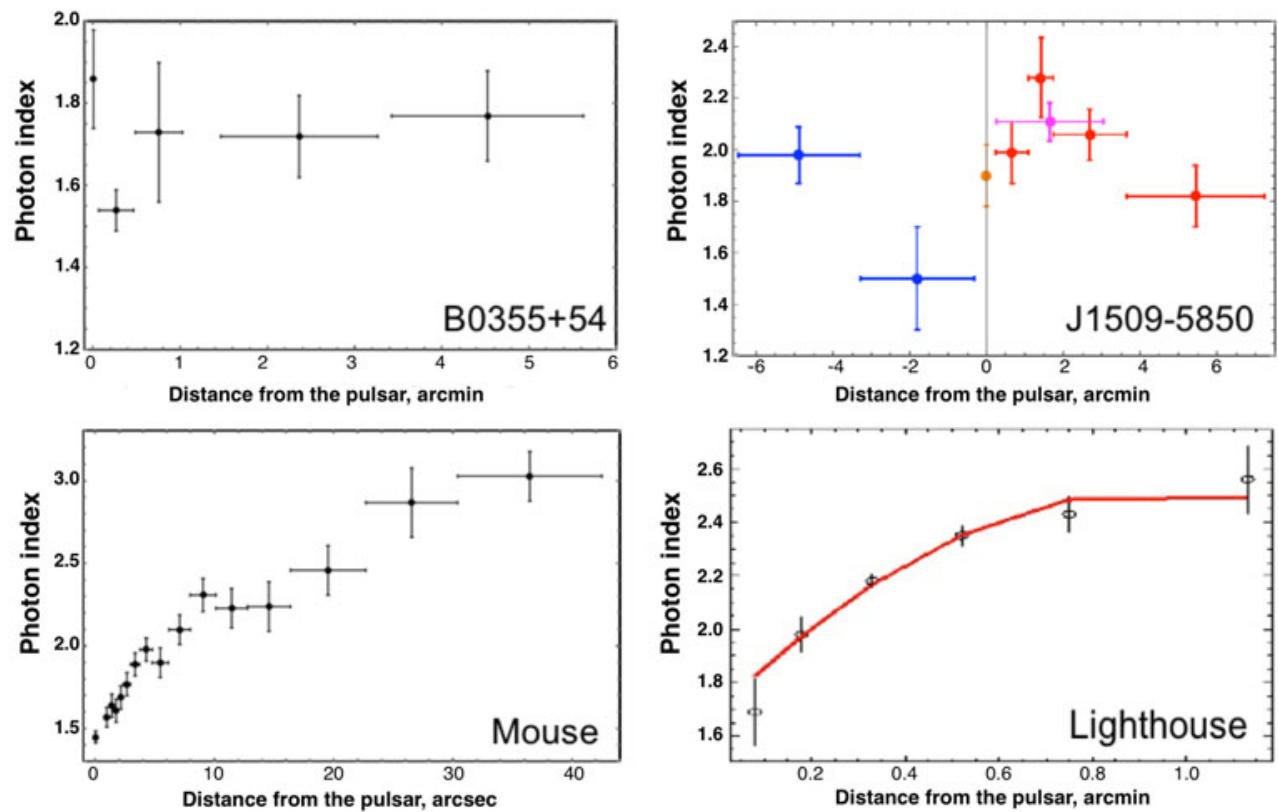

FIGURE 8. Variation of spectral slopes along pulsar tails. No cooling trends are seen in B0355+54 and J1509-5850, while cooling (spectral softening) is very pronounced in the Mouse (Klingler et al., in prep.) and Lighthouse (Pavan et al. 2016), perhaps due to higher magnetic fields, lack of in-situ acceleration or slower flow speed. The red line in the Lighthouse panel shows the best fit with a parabolic function (from Pavan et al. 2016).

Another puzzling property is the apparent faintness of pulsar tails in $\mathrm{TeV}^{10}$ while inverse-Compton $\mathrm{TeV}$ emission has been detected from many younger, more compact PWNe (see e.g. Kargaltsev, Rangelov \& Pavlov (2013) for a review). In the leptonic scenario (inverse-Compton up-scattering of cosmic microwave background radiation, dust infrared (IR) emission, and starlight photons off relativistic electrons and positrons), the PWN TeV luminosity should not depend on particle density of the surrounding medium. The lack of detections can be attributed to the limitations of the current $\mathrm{TeV}$ observatories that have poor angular resolution and may not be sensitive to narrow long structures such as pulsar tails ${ }^{11}$.

Yet another puzzle of pulsar tails is the very different dependences of their X-ray spectra on the distance from the pulsar along the tail (see figure 8). The rapid changes of photon index, likely due to synchrotron cooling, are seen in the tails of younger pulsars (e.g. J1747-2958, J1101-6101, and J0537-6910) while virtually no changes are seen in the tails of older pulsars (e.g. J1509-5850 and B0355+54). The different spectral evolution might be explained by different strength of magnetic field. The magnetic field strengths could be reduced by more efficient reconnection in the tail in the case of pulsars with a large angle between the spin axis and the velocity vector because larger angles may lead to a more tangled large-scale magnetic field in the

\footnotetext{
${ }^{10} \mathrm{TeV}$ emission has been recently detected from Geminga, but it is likely produced in a large-scale 'halo' rather than in the tail (see Abeysekara et al. 2017 and Linden et al. 2017).

${ }^{11}$ The sizes of circular extraction regions typically used in $\mathrm{TeV}$ data analysis substantially exceed the transverse sizes of the tails due to the coarse resolution of current $\mathrm{TeV}$ imaging arrays. Under these circumstances the faint $\mathrm{TeV}$ emission from the tail could be buried in the background.
} 
tail. The continuing reconnection of magnetic fields in long tails could also lead to particle re-acceleration, which could help to explain the lack of softening in the X-ray spectra of the J1509-5850 and B0355+54 tails. In cases where the spin axis is nearly aligned with the magnetic dipole axis, reconnection may be delayed (because magnetic energy is not being efficiently converted to particle energy) until significant distortions or turbulence develop at larger distances down the tail (which may explain the lack of a 'head' in the X-ray images of J0357+3205). A larger sample of bow shock PWNe is needed to probe these scenarios.

From (3.4) in $\S 3$, it is possible to crudely estimate the average characteristic flow speed in the tail: $v_{\text {flow }} \sim l / t_{\text {syn }}$, where $l$ is the length of the $\mathrm{X}$-ray tail. The bulk flow speeds estimated in this simplistic way are $2000,3000,3000$, and $>1000 \mathrm{~km} \mathrm{~s}^{-1}$ in the B0355+54, J1509-5850, J1101-6101 and the Mouse tails, respectively ${ }^{12}$. They are significantly higher that the pulsar speeds (with a possible exception of the very fast PSR J1101-6101).

\section{Misaligned outflows}

In recent deep X-ray observations, a new type of structure has unexpectedly been discovered in some supersonic PWNe. Extended, elongated features, strongly misaligned with the pulsar's direction of motion, are seen originating from the vicinity of four pulsars (see figure 9): the Guitar Nebula (PSR B2224+65; Hui \& Becker 2007), the Lighthouse PWN (PSR J1101-6101; Pavan et al. 2014, 2016), the PWN of PSR J1509-5058 (Klingler et al. 2016a), and the Mushroom PWN of PSR B0355+54 (Klingler et al. 2016b). Another, possibly similar, misaligned feature was reported for PSR J2055+2539 based on XMM-Newton observations (Marelli et al. 2016b). The misaligned orientation of these features is puzzling because, for a fast-moving pulsar, one would expect all of the PW to be confined within the tail (which three of the four PWNe exhibit as well). In principle, one could imagine a highly anisotropic wind with extremely strong polar outflows (jets) misaligned with the pulsar's velocity vector. Such jets should be bent by the ram pressure of the oncoming ISM, with the bending length scale $l_{b} \sim \xi_{j} \dot{E} /\left(c r_{j} \rho v^{2} \sin \theta\right)$, where $\xi_{j}<1$ is the fraction of the spin-down power $\dot{E}$ that goes into the jet, $r_{j}$ is the jet radius, and $\theta$ is the angle between the initial jet direction and the pulsar's velocity. However, the bending length-scales are way too small compared to the lengths of the nearly straight misaligned features. For instance, $l_{b} \sim 0.065 \xi_{j}\left(r_{j} / 5.7 \times 10^{16} \mathrm{~cm}\right)^{-1} n^{-1}(\sin \theta)^{-1}\left(v / 300 \mathrm{~km} \mathrm{~s}^{-1}\right)^{-2} \mathrm{pc}$ for J1509-5850, while the observed length of the misaligned outflow is $\sim 8$ pc (for $d=3.8 \mathrm{kpc}$; see Klingler et al. $2016 a$ for details). Therefore, it is difficult to explain the misaligned outflows with a hydrodynamic-type model.

To interpret the first discovered misaligned outflow in the Guitar Nebula, Bandiera (2008) suggested that such structures can be formed in high- $\mathcal{M}$ pulsars when the gyro-radii of most energetic electrons, $r_{g}=\gamma m_{e} c^{2} / e B_{\text {apex }}$, are comparable to or exceed the stand-off distance of the bow shock apex, $R_{h}=\left(\dot{E} / 4 \pi c m_{p} n v^{2}\right)^{1 / 2}$ (for isotropic wind), where $B_{\text {apex }}$ is the magnetic field inside the PWN (between the contact discontinuity and termination shock) near the bow shock apex. Such particles cannot be contained within the bow shock, and can hence 'leak' into the ISM, where they diffuse along the ambient ISM magnetic field lines and radiate synchrotron photons. The Lorentz factors of escaping electrons, therefore,

\footnotetext{
${ }^{12}$ An additional uncertainty in these estimates comes from the unknown inclination angle of the velocity with respect to the line of sight. For these estimates we used the values that were considered plausible in the corresponding individual publications (see table 2).
} 


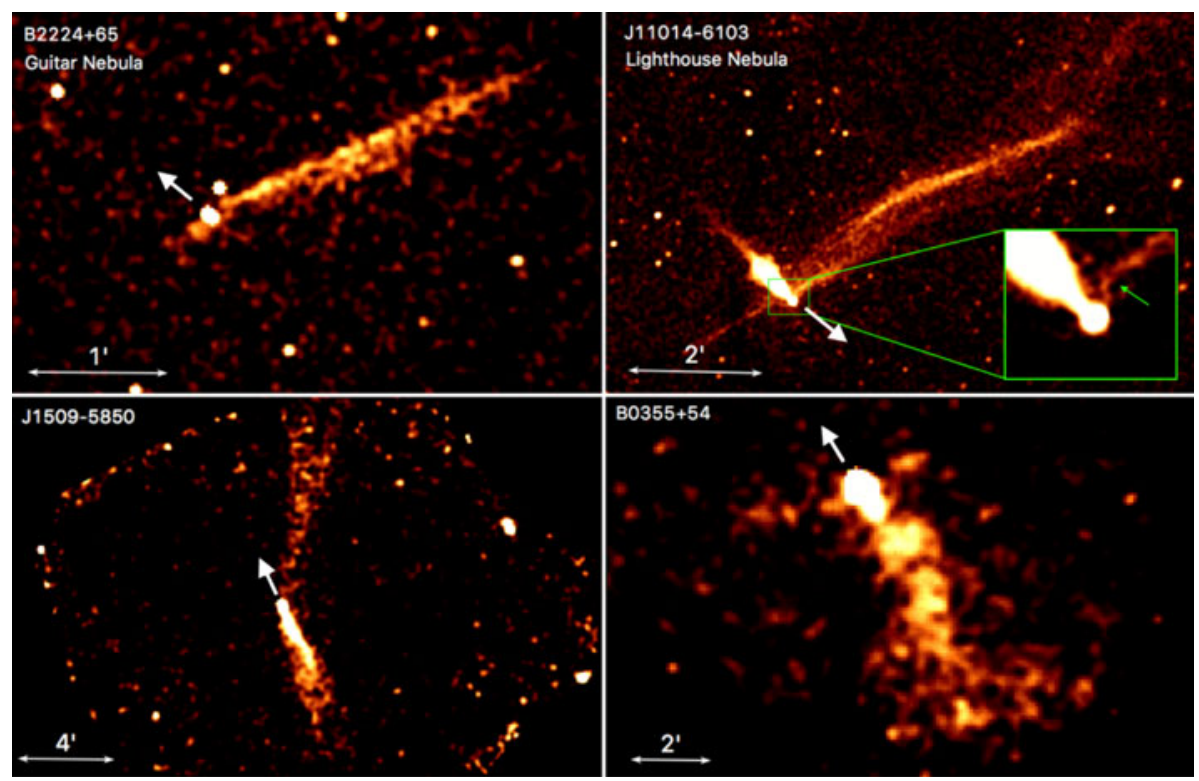

FIGURE 9. Chandra images of PWNe displaying misaligned outflows. The white arrows show the directions of pulsar proper motion, and the green arrow shows the bending in the Lighthouse Nebula outflow (inset). Chandra images of some of these objects are also shown in Reynolds et al. (2017).

can be estimated as $\gamma \sim 2 \times 10^{8}(E / 1 \mathrm{keV})^{1 / 2}\left(B_{\mathrm{ISM}} / 5 \mu \mathrm{G}\right)^{-1 / 2}$, where $B_{\mathrm{ISM}}$ is the ambient magnetic field, and $E$ is the synchrotron photon energy that reaches at least $8 \mathrm{keV}$ for the Lighthouse, Guitar and J1509-5058 PWNe. From the escape condition, $r_{g} \gtrsim R_{h}$, one can set an upper limit on the PWN field near the apex: $B_{\text {apex }} \lesssim 34(E / 1 \mathrm{keV})^{1 / 2}\left(B_{\mathrm{ISM}} / 5 \mu \mathrm{G}\right)^{-1 / 2}\left(R_{h} / 10^{16} \mathrm{~cm}\right)^{-1} \mu \mathrm{G}$, if $R_{h}$ is directly measured or estimated from the equation given above.

It is important to stress that in this scenario the misaligned outflows are an entirely kinetic phenomenon, which makes them very different from the pressure-confined jets of tails. The observed appearances of the misaligned outflows should reflect the ambient ISM structure ${ }^{13}$ illuminated by synchrotron emission from the leaked PW particles. In the discussed scenario, the misaligned structures are expected to move with the particle injection site (SPWN apex) which moves with the pulsar.

The Bandiera (2008) scenario was challenged by recent deep Chandra observations of the Lighthouse PWN where the misaligned outflow appears to bend around the pulsar (as indicated by the green arrow in the inset image in the top right panel of figure 9). Although the distortion of the external (ISM) field can be expected if a magnetized object is moving through the ISM ('magnetic draping'; Lyutikov 2006 and Dursi \& Pfrommer 2008), this should not be happening outside the forward shock region where the ISM 'does not know' about the incoming object yet. Another difficulty with this scenario is that most of these misaligned outflows (with a possible exception of a very faint one associated with B0355+54) are strongly asymmetric (i.e. they appear much brighter and longer on one side of the pulsar).

\footnotetext{
${ }^{13}$ If the leaking particles carry sufficiently large currents, the currents can perturb the original ambient ISM magnetic field.
} 


\section{Forward shock emission}

If the local ISM contains neutral Hydrogen, collisional excitation and charge exchange in the forward shock can cause the shocked ISM to emit in $H \alpha$ leading to formation of an $H \alpha$ bow shock. To date, bow-shaped $H \alpha$ nebulae have been detected around only eight rotation-powered pulsars (see table 2 and Brownsberger \& Romani 2014). A possible explanation for the scarcity of $H \alpha$ bow shocks is a high degree of ionization of the ambient medium ahead of the pulsar, which might be caused by pre-ionization of the ISM by radiation from the pulsar and/or the X-ray PWN.

One of the first $H \alpha$ pulsar bow shocks was detected by Bell, Bailes \& Bessell (1993) ahead of the binary millisecond (recycled) pulsar J0437-4715. PSR J04374715 is the closest known pulsar, $d=156.8 \pm 0.2$ pc (Reardon et al. 2016), with an accurately measured proper motion corresponding to the transverse velocity $v_{\perp}=$ $104.1 \pm 0.2 \mathrm{~km} \mathrm{~s}^{-1}$ (Deller et al. 2008). It has a period of $5.8 \mathrm{~ms}$ and a spin-down power $\dot{E}=2.9 \times 10^{33} I_{45} \mathrm{erg} \mathrm{s}^{-1}$ (corrected for the Shklovskii effect; $I_{45}$ is the neutron star moment of inertia in units of $10^{45} \mathrm{~g} \mathrm{~cm}^{2}$ ). Its $H \alpha$ bow shock shows a symmetric structure with a stand-off distance of approximately $10^{\prime \prime}\left(2.3 \times 10^{16} \mathrm{~cm}\right)$ between the pulsar and the bow shock apex (see figure 10).

The J0437-4715 bow shock is the first one detected in far-UV, thanks to the pulsar proximity and deep observations with the Hubble Space Telescope. The far-UV images reveal a bow-like structure positionally coincident with the $H \alpha$ bow shock (figure 10). The measurements in broad F125LP and F140LP filters (wavelength bands are 1250-2000 $\AA$ and 1350-2000 $\AA$, respectively) do not allow us to establish the spectral model. The existing data are consistent with both a simple power-law spectrum, which could be continuum synchrotron emission from relativistic electrons trapped in the forward shock region (Bykov et al. 2017), and the collisionless plasma emission (spectral lines plus continuum) from the ISM matter compressed and heated in the forward shock region (Bykov et al. 2013).

Analysing a subset of archival Chandra ACIS data, Rangelov et al. (2016) also found a faint extended X-ray emission ahead of the pulsar but well within the $H \alpha$ bow shock (figure 10). This emission was tentatively interpreted as an X-ray PWN of J0437. The morphology of the PWN is unusual compared to other supersonic PWNe in the sense that the X-ray emission is seen only ahead of the moving pulsar, being confined to a relatively narrow region. However, such a bright region of emission ahead of the pulsar consistent with simulated synchrotron maps of the bow shock PWNe (see figure 4 from Bucciantini et al. 2005). These regions may simply be unresolvable in other, more remote PWNe, but due to the proximity of J0437, Chandra observations can probe shocked PW X-ray emission on much smaller spatial scales.

The second bow shock detected in far-UV is associated with the solitary millisecond PSR J2124-3358 (Rangelov et al. 2017). This pulsar has a period of $4.93 \mathrm{~ms}$ and a spin-down power $\dot{E}=6.8 \times 10^{33} I_{45} \mathrm{erg} \mathrm{s}^{-1}$. It is at a distance $d=410_{-70}^{+90} \mathrm{pc}$, its proper motion corresponds to $v_{\perp}=101.2 \pm 0.8 \mathrm{~km}$, at $d=410 \mathrm{pc}$ (Reardon et al. 2016). The shape of its far-UV bow shock matches well the $H \alpha$ bow shock, which suggests that the far-UV emission comes from the forward shock region. The poor statistics once again prevents us from discriminating between the power-law and collisionless shock plasma emission models. A sensitive slit or integral field spectroscopy would enable further progress in understanding the nature of far-UV emission from the pulsar bow shocks.

Hui \& Becker (2006) reported an X-ray PWN associated with J2124-3358. It was detected with both XMM-Newton and Chandra, but only Chandra was able to resolve the nebula into a one-sided narrow structure, which appears to be projected inside the 
(a)

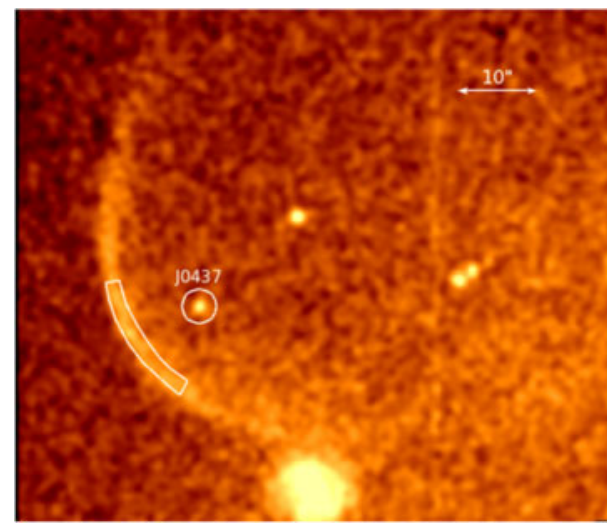

(c)

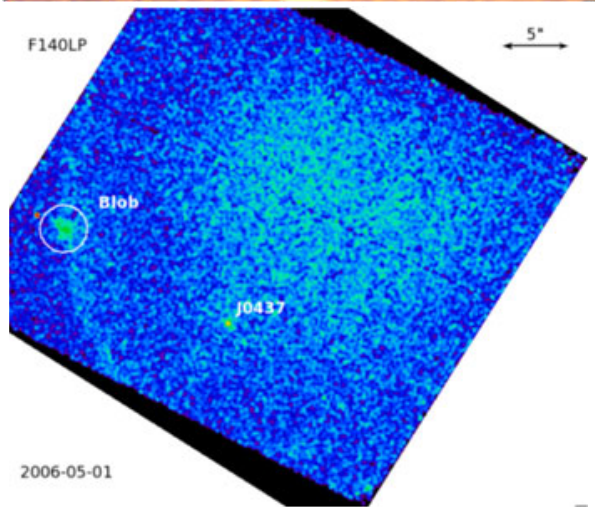

(b)

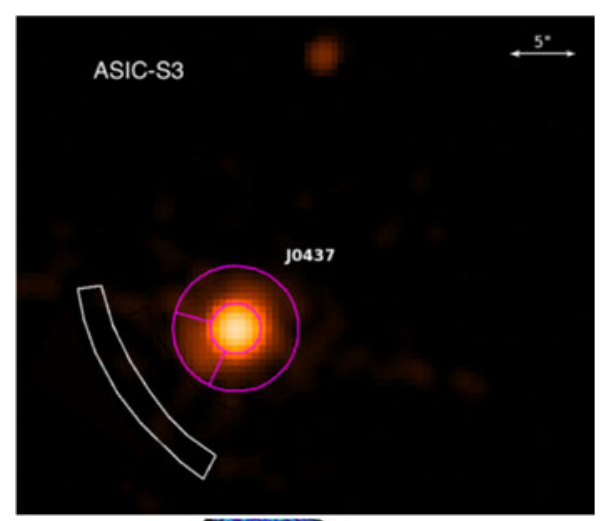

$(d)$

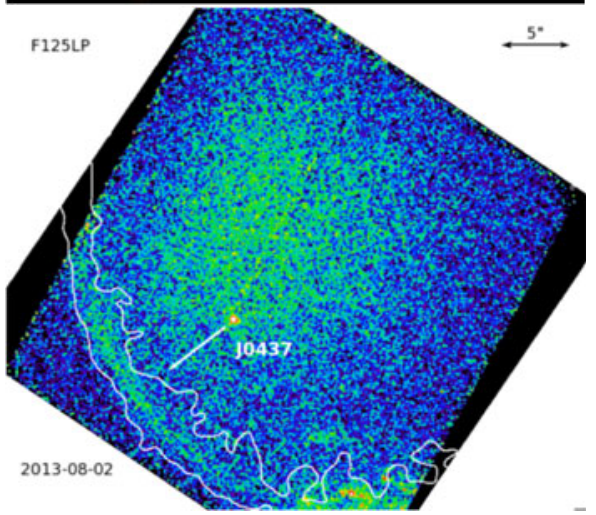

FIGURE 10. (a) Shows an $H \alpha$ image of PSR J0437-4715 obtained with the SOAR telescope (Brownsberger \& Romani 2014). (b) Shows a Chandra ACIS-S3 image. The magenta contours delineate the source and background extraction regions used for the extended emission analysis; the white contour, coinciding with that in $(a)$, shows the position of the $H \alpha$ bow shock apex. $(c, d)$ Show far-UV images from two different observations (dates are labelled) and different filters (also labelled). The non-uniform background prominent in the far-UV images is due to the 'thermal glow' of the detector. The extended 'blob' of unknown nature is seen in $(c) . H \alpha$ bow shock contours (based on the SOAR image) are shown in $(d)$. North is up, East is to the left. The images are taken from Rangelov et al. (2016).

observed $H \alpha$-far-UV bow shock (see figure 11). The X-ray emission extends northwest from the pulsar for $\sim 0.5$. Hui \& Becker (2006) modelled the spectrum of the emission with a power-law model with photon index $\Gamma=2.2 \pm 0.4$.

In addition to eight rotation-powered pulsars, bow-shaped $H \alpha$ emission has been detected around the radio-quiet isolated neutron star RX J1856.5-3754 (van Kerkwijk \& Kulkarni 2001). It belongs to the class of neutron stars that do not show any signatures of magnetospheric activity (e.g. non-thermal X-ray emission or radio/ $\gamma$-ray pulsations; see Kaplan et al. (2011) and references therein). In this case the $H \alpha$ emission may represent a photoionization nebula rather than a bow shock (van Kerkwijk \& Kulkarni 2001). For this reason we do not include RX J1856.5-3754 in tables 1 and 2 . 
(a)

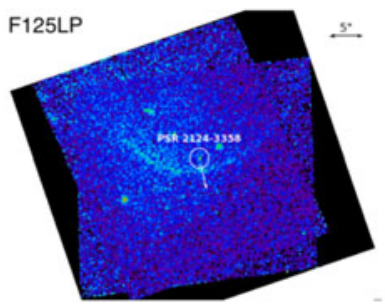

(b)

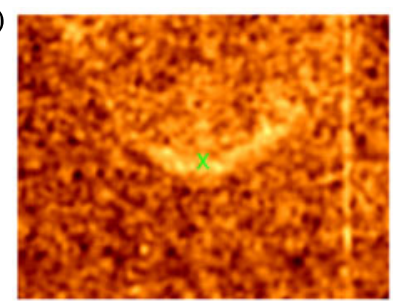

(c)

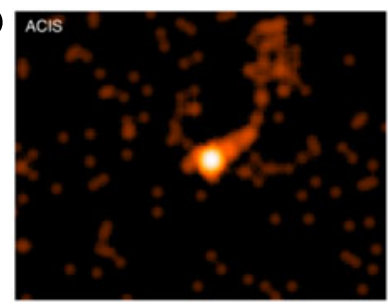

FIgURE 11. (a) Shows a far-UV image of the PSR J2124-3358 vicinity obtained with the Hubble Space Telescope ACS/SBC F125LP filter. The pulsar and its direction of motion are shown with white circle and arrow. $(b)$ Shows the SOAR $H \alpha$ image; ' $\mathrm{X}$ ' marks the position of the pulsar. (c) Shows the Chandra ACIS image in 0.5-7 keV. All images are to the same scale. North is up, and East to the left. The images are taken from Rangelov et al. (2017).

\section{Conclusions}

SPWNe exhibit a number of interesting properties providing insight into the structures of neutron star magnetospheres, winds and pulsar viewing geometry, as well as into the physics of collisionless shocks and supernova explosions. Some of these properties became apparent only from deep Chandra and Hubble Space Telescope observations:

(i) Very long (up to $8 \mathrm{pc}$ ) X-ray tails have been detected behind a few pulsars. In some cases the $\mathrm{X}$-ray tails are accompanied by even longer radio tails. None of these long tails are seen in $\mathrm{TeV}$.

(ii) Some of the tails exhibit very rapid spectral softening (cooling) with increasing distance from the pulsar (e.g. the Mouse and Lighthouse PWNe), while others show no evidence of cooling (e.g. J1509-5850).

(iii) When resolved in X-rays, the SPWN head morphologies can differ drastically. There can be 'filled', 'empty', and very dim (of absent) SPWN heads. The differences can be linked to the geometry of the pulsar magnetosphere and to the orientation of pulsar's spin axis with respect to the observer's line of sight.

(iv) There is a growing number of SPWNe with misaligned outflows which are likely produced by most energetic particles leaking from the SPWN apex. However, there are some unsolved puzzles in this scenario.

(v) Far-UV emission from two $H \alpha$ bow shocks has been recently discovered, likely produced by heated shocked ISM.

Although a general, qualitative picture of PWNe created by supersonically moving pulsars is more or less clear, there remain a number of open questions.

(i) Why are some SPWN heads very faint in X-rays? Are they simply too compact to be resolved from the pulsar?

(ii) Why do X-ray spectra of some tails remain hard at large distances from the pulsar?

(iii) What are particle acceleration mechanisms in SPWNe, and is there in-situ acceleration outside the termination shock?

(iv) How some of the tails remain so well collimated for such long distances?

(v) What is the nature of the misaligned outflows? Why are they so asymmetric? 
(vi) Are the morphologies of X-ray PWN heads, PWN radiative efficiencies, and PWN spectra uniquely determined by mutual orientations of the line of sight, pulsar's velocity, spin and magnetic axis?

(vii) Are the SPWNe intrinsically fainter in $\mathrm{TeV} \gamma$-rays compared to $\mathrm{PWNe}$ inside the SNRs?

To answer these and other questions, detailed studies of a larger sample of SPWNe must be supplemented by more realistic models.

\section{Acknowledgements}

This work was partly supported by the NASA through Chandra Awards GO3-14057 and G03-14082 issued by the Chandra X-ray Observatory Center, which is operated by the Smithsonian Astrophysical Observatory for and on behalf of the NASA under contract NAS8-03060. It was also supported by grants GO-12917 and GO-13783 from the Space Telescope Science Institute, which is operated by Association of Universities for Research in Astronomy, Inc. under NASA contract NAS 5-26555. We thank I. Volkov for discussions and help with the figures.

\section{REFERENCES}

Abeysekara, A. U., Albert, A., Alfaro, R., Alvarez, C., Álvarez, J. D., Arceo, R., Arteaga-Velázquez, J. C., Ayala Solares, H. A., Barber, A. S., Baughman, B. et al. 2017 The 2HWC HAWC observatory gamma-ray catalog. Astrophys. J. 843, 40.

Acciari, V. A., Aliu, E., Arlen, T., Aune, T., Bautista, M., Beilicke, M., Benbow, W., Bradbury, S. M., Buckley, J. H., Bugaev, V. et al. 2009 Observation of extended very high energy emission from the supernova remnant IC 443 with VERITAS. Astrophys. J. Lett. 698, L133-L137.

ACERO, F. 2011 Detection of TeV emission from the intriguing composite SNR G327.1-1.1. Intl Cosmic Ray Conf. 7, 185.

Aharonian, F., Akhperjanian, A., Beilicke, M., Bernlöhr, K., Börst, H.-G., Bojahr, H., Bolz, O., Coarasa, T., Contreras, J. L., Cortina, J. et al. 2004 The crab nebula and pulsar between $500 \mathrm{GeV}$ and $80 \mathrm{TeV}$ : observations with the HEGRA stereoscopic air cerenkov telescopes. Astrophys. J. 614, 897-913.

Auchettl, K., Slane, P., Romani, R. W., Posselt, B., Pavlov, G. G., Kargaltsev, O., Ng, C.-Y., Temim, T., Weisskopf, M. C., BYKov, A. et al. 2015 X-ray analysis of the proper motion and pulsar wind nebula for PSR J1741-2054. Astrophys. J. 802, 68.

BANDIERA, R. 2008 On the X-ray feature associated with the Guitar nebula. Astron. Astrophys. 490, L3-L6.

Becker, W., Kramer, M., Jessner, A., TaAm, R. E., Jia, J. J., Cheng, K. S., Mignani, R., Pellizzoni, A., De LuCA, A., SŁowiKowsKa, A. et al. 2006 A multiwavelength study of the pulsar PSR B1929+10 and its X-ray trail. Astrophys. J. 645, 1421-1435.

Bell, J. F., BAiles, M. \& Bessell, M. S. 1993 Optical detection of the companion of the millisecond pulsar J0437-4715. Nature 364, 603-605.

Blazek, J. A., Gaensler, B. M., Chatterjee, S., van der Swaluw, E., Camilo, F. \& STAPPERS, B. W. 2006 The duck redux: an improved proper-motion upper limit for the pulsar B1757-24 near the supernova remnant G5.4-1.2. Astrophys. J. 652, 1523-1530.

Brownsberger, S. \& Romani, R. W. 2014 A survey for $H \alpha$ pulsar bow shocks. Astrophys. J. 784, 154.

Bucciantini, N., Amato, E. \& Del Zanna, L. 2005 Relativistic MHD simulations of pulsar bow-shock nebulae. Astron. Astrophys. 434, 189-199.

BüHLER, R. \& BLANDFORD, R. 2014 The surprising crab pulsar and its nebula: a review. Rep. Prog. Phys. 77 (6), 066901. 
BÜHLER, R. \& GIOMI, M. 2016 The imprint of pulsar parameters on the morphology of pulsar wind nebulae. Mon. Not. R. Astron. Soc. 462, 2762-2776.

Bykov, A. M., Amato, E., Petrov, A. E., Krassilchtchikov, A. M. \& Levenfish, K. P. 2017 Pulsar wind nebulae with bow shocks: non-thermal radiation and cosmic ray leptons. Space Sci. Rev. 207, 235-290.

Bykov, A. M., Malkov, M. A., Raymond, J. C., Krassilchtchikov, A. M. \& Vladimirov, A. E. 2013 Collisionless shocks in partly ionized plasma with cosmic rays: microphysics of non-thermal components. Space Sci. Rev. 178, 599-632.

Camilo, F., NG, C.-Y., Gaensler, B. M., Ransom, S. M., Chatterjee, S., Reynolds, J. \& SARKISSIAN, J. 2009 Out of the frying pan: a young pulsar with a long radio trail emerging from SNR G315.9-0.0. Astrophys. J. Lett. 703, L55-L58.

Caraveo, P. A., Bignami, G. F., De Luca, A., Mereghetti, S., Pellizzoni, A., Mignani, R., TUR, A. \& BECKER, W. 2003 Geminga's tails: a pulsar bow shock probing the interstellar medium. Science 301, 1345-1348.

Chatterjee, S., Brisken, W. F., Vlemmings, W. H. T., Goss, W. M., Lazio, T. J. W., Cordes, J. M., Thorsett, S. E., Fomalont, E. B., Lyne, A. G. \& Kramer, M. 2009 Precision astrometry with the very long baseline array: parallaxes and proper motions for 14 pulsars. Astrophys. J. 698, 250-265.

De luca, A., Marelli, M., Mignani, R. P., Caraveo, P. A., Hummel, W., Collins, S., Shearer, A., Saz Parkinson, P. M., Belfiore, A. \& Bignami, G. F. 2011 Discovery of a faint X-ray counterpart and a parsec-long X-ray tail for the middle-aged, $\gamma$-ray-only pulsar PSR J0357+3205. Astrophys. J. 733, 104.

De luca, A., Mignani, R. P., Marelli, M., Salvetti, D., Sartore, N., Belfiore, A., Saz Parkinson, P., Caraveo, P. A. \& Bignami, G. F. 2013 PSR J0357+3205: a fast-moving pulsar with a very unusual X-ray trail. Astrophys. J. Lett. 765, L19.

Deller, A. T., Verbiest, J. P. W., Tingay, S. J. \& Bailes, M. 2008 Extremely high precision VLBI astrometry of PSR J0437-4715 and implications for theories of gravity. Astrophys. $J$. Lett. 685, L67.

Dursi, L. J. \& Pfrommer, C. 2008 Draping of cluster magnetic fields over bullets and bubbles morphology and dynamic effects. Astrophys. J. 677, 993-1018.

Frail, D. A., Giacani, E. B., Goss, W. M. \& Dubner, G. 1996 The pulsar wind nebula around PSR B1853+01 in the supernova remnant W44. Astrophys. J. Lett. 464, L165.

Gaensler, B. M., Chatterjee, S., Slane, P. O., van der Swaluw, E., Camilo, F. \& Hughes, J. P. 2006 The X-ray structure of the pulsar bow shock G189.22+2.90 in the supernova remnant IC 443. Astrophys. J. 648, 1037-1042.

Gaensler, B. M., Stappers, B. W., Frail, D. A. \& Johnston, S. 1998 An unusual pulsar wind nebula associated with PSR B0906-49. Astrophys. J. Lett. 499, L69-L73.

Gaensler, B. M., van der Swaluw, E., Camilo, F., Kaspi, V. M., Baganoff, F. K., YusefZADEh, F. \& MANChESTER, R. N. 2004 The mouse that soared: high-resolution X-ray imaging of the pulsar-powered bow shock G359.23-0.82. Astrophys. J. 616, 383-402.

Ginzburg, V. L. \& SyrovatskiI, S. I. 1964 The Origin of Cosmic Rays. Pergamon.

Grondin, M.-H., Romani, R. W., Lemoine-Goumard, M., Guillemot, L., Harding, A. K. \& REPoseur, T. 2013 The Vela-X pulsar wind nebula revisited with four years of Fermi large area telescope observations. Astrophys. J. 774, 110.

Hales, C. A., Gaensler, B. M., Chatterjee, S., van der Swaluw, E. \& Camilo, F. 2009 A proper motion for the pulsar wind nebula G359.23-0.82, the 'mouse,' associated with the energetic radio pulsar J1747-2958. Astrophys. J. 706, 1316-1322.

Halpern, J. P., Tomsick, J. A., Gotthelf, E. V., Camilo, F., NG, C.-Y., Bodaghee, A., Rodriguez, J., Chaty, S. \& Rahoui, F. 2014 Discovery of X-ray pulsations from the INTEGRAL source IGR J11014-6103. Astrophys. J. Lett. 795, L27.

Hobis, G., Lorimer, D. R., Lyne, A. G. \& Kramer, M. 2005 A statistical study of 233 pulsar proper motions. Mon. Not. R. Astron. Soc. 360, 974-992.

Huang, R. H. H., Kong, A. K. H., Takata, J., Hui, C. Y., Lin, L. C. C. \& Cheng, K. S. 2012 X-ray studies of the black widow pulsar PSR B1957+20. Astrophys. J. 760, 92.

Hui, C. Y. \& BECKER, W. 2006 Searches for diffuse X-ray emission around millisecond pulsars: an X-ray nebula associated with PSR J2124-3358. Astron. Astrophys. 448, L13-L17. 
Hui, C. Y. \& BeCKeR, W. 2007 X-ray emission properties of the old pulsar PSR B2224+65. Astron. Astrophys. 467, 1209-1214.

Hui, C. Y., Huang, R. H. H., Trepl, L., Tetzlaff, N., Takata, J., Wu, E. M. H. \& Cheng, K. S. 2012 XMM-Newton observation of PSR B2224+65 and its jet. Astrophys. J. 747, 74.

Kaplan, D. L., Kamble, A., van KerkwiJk, M. H. \& Ho, W. C. G. 2011 New optical/ultraviolet counterparts and the spectral energy distributions of nearby, thermally emitting, isolated Neutron stars. Astrophys. J. 736, 117.

Kargaltsev, O., Cerutti, B., Lyubarsky, Y. \& Striani, E. 2015 Pulsar-wind nebulae. Recent progress in observations and theory. Space Sci. Rev. 191, 391-439.

Kargaltsev, O., Durant, M., Pavlov, G. G. \& Garmire, G. 2012 Chandra Pulsar Survey (ChaPS). Astrophys. J. Suppl. 201, 37.

Kargaltsev, O., Misanovic, Z., Pavlov, G. G., Wong, J. A. \& Garmire, G. P. 2008 X-ray observations of parsec-scale tails behind two middle-aged pulsars. Astrophys. J. 684, 542-557.

Kargaltsev, O.\& PaVlov, G. G. 2007 X-ray emission from PSR J1809-1917 and its pulsar wind nebula, possibly associated with the TeV gamma-ray source HESS J1809-193. Astrophys. $J$. 670, 655-667.

Kargaltsev, O.\& Pavlov, G. G. 2008 Pulsar wind nebulae in the Chandra era. In 40 Years of Pulsars: Millisecond Pulsars, Magnetars and More (ed. C. Bassa, Z. Wang, A. Cumming \& V. M. Kaspi), American Institute of Physics Conference Series, vol. 983, pp. 171-185. American Institute of Physics.

KargaltseV, O. \& PaVlov, G. G. 2010 Pulsar-wind nebulae in X-rays and TeV $\gamma$-rays. In $X$-ray Astronomy 2009; Present Status, Multi-Wavelength Approach and Future Perspectives, vol. 1248, pp. 25-28. American Institute of Physics.

Kargaltsev, O., Rangelov, B. \& Pavlov, G. 2013 Pulsar-Wind Nebulae as a Dominant Population of Galactic VHE Sources, pp. 359-406. Nova Science Publishers.

Kaspi, V. M., Gotthelf, E. V., Gaensler, B. M. \& Lyutikov, M. 2001 X-ray detection of pulsar PSR B1757-24 and its nebular tail. Astrophys. J. Lett. 562, L163-L166.

Klingler, N., Kargaltsev, O., Rangelov, B., Pavlov, G. G., Posselt, B. \& NG, C.-Y. $2016 a$ Chandra observations of outflows from PSR J1509-5850. Astrophys. J. 828, 70.

Klingler, N., Rangelov, B., Kargaltsev, O., Pavlov, G. G., Romani, R. W., Posselt, B., Slane, P., Temim, T., NG, C.-Y., Bucciantini, N. et al. 2016b Deep Chandra observations of the pulsar wind nebula created by PSR B0355+54. Astrophys. J. 833, 253.

Komissarov, S. S. \& LYUBARSKY, Y. E. 2004 Synchrotron nebulae created by anisotropic magnetized pulsar winds. Mon. Not. R. Astron. Soc. 349, 779-792.

Kramer, M. \& Johnston, S. 2008 High-precision geometry of a double-pole pulsar. Mon. Not. R. Astron. Soc. 390, 87-92.

KUIPER, L. \& HERMSEN, W. 2015 The soft $\gamma$-ray pulsar population: a high-energy overview. Mon. Not. R. Astron. Soc. 449, 3827-3866.

Levenfish, K. P., Bykov, A. M., Durant, M., Kargaltsev, O. Y., Kropotina, Y. A., Pavlov, G. G., Krassilchtchikov, A. M. \& Uvarov, Y. A. 2013 Finest persistent structures in the Vela PWN. Memorie della Societa Astronomica Italiana 84, 588.

Linden, T., Auchettl, K., Bramante, J., Cholis, I., Fang, K., Hooper, D., Karwal, T. \& WeIsHI LI, S. 2017 Using HAWC to discover invisible pulsars. ArXiv e-prints: arXiv:1703.09704.

LyUbARSKy, Y. \& KiRK, J. G. 2001 Reconnection in a striped pulsar wind. Astrophys. J. 547, 437-448.

LyUtikov, M. 2006 Magnetic draping of merging cores and radio bubbles in clusters of galaxies. Mon. Not. R. Astron. Soc. 373, 73-78.

Ma, Y. K., NG, C.-Y., Bucciantini, N., Slane, P. O., Gaensler, B. M. \& Temim, T. 2016 Radio polarization observations of the snail: a crushed pulsar wind nebula in G327.1-1.1 with a highly ordered magnetic field. Astrophys. J. 820, 100.

Manchester, R. N., Hobbs, G. B., TeOH, A. \& Hobbs, M. 2005 The Australia telescope national facility pulsar catalogue. Astron. J. 129, 1993-2006.

Marelli, M., Mignani, R. P., De Luca, A., Saz Parkinson, P. M., Salvetti, D., Den HaRtog, P. R. \& WolfF, M. T. 2015 Radio-quiet and radio-loud pulsars: similar in gammarays but different in X-rays. Astrophys. J. 802, 78. 
Marelli, M., Pizzocaro, D., De luca, A., Gastaldello, F., Caraveo, P. \& Saz Parkinson, P. $2016 a$ The tale of the two tails of the Oldish PSR J2055+2539. Astrophys. J. 819, 40.

Marelli, M., Pizzocaro, D., De luca, A., Gastaldello, F., Caraveo, P. \& Saz Parkinson, P. $2016 b$ The tale of the two tails of the Oldish PSR J2055+2539. Astrophys. J. 819, 40.

Mattana, F., Götz, D., Terrier, R., Bouchet, L., Ponti, G., Falanga, M., Renaud, M., Caballero, I., Soldi, S., Zurita Heras, J. A. et al. 2011 Extended hard X-ray emission from the Vela pulsar wind nebula. Astrophys. J. Lett. 743, L18.

McGowan, K. E., Vestrand, W. T., Kennea, J. A., Zane, S., Cropper, M. \& Córdova, F. A. 2006 Probing the pulsar wind nebula of PSR B0355+54. Astrophys. J. 647, 1300-1308.

Misanovic, Z., Pavlov, G. G. \& Garmire, G. P. 2008 Chandra observations of the pulsar B1929+10 and its environment. Astrophys. J. 685, 1129-1142.

Moon, D.-S., Lee, J.-J., Eikenberry, S. S., Koo, B.-C., Chatterjee, S., Kaplan, D. L., Hester, J. J., Cordes, J. M., Gallant, Y. A. \& Koch-Miramond, L. 2004 PSR B1951+32: a bow shock-confined X-ray nebula, a synchrotron knot, and an optical counterpart candidate. Astrophys. J. Lett. 610, L33-L36.

Morlino, G., LyUtikOV, M. \& Vorster, M. 2015 Mass loading of bow shock pulsar wind nebulae. Mon. Not. R. Astron. Soc. 454, 3886-3901.

NG, C.-Y., Bucciantini, N., Gaensler, B. M., Camilo, F., Chatterjee, S. \& Bouchard, A. 2012 An extreme pulsar tail protruding from the frying pan supernova remnant. Astrophys. $J$. 746, 105.

Ng, C.-Y., Gaensler, B. M., Chatterjee, S. \& Johnston, S. 2010 Radio polarization observations of G319.9-0.7: a bow-shock nebula with an azimuthal magnetic field powered by pulsar J1509-5850. Astrophys. J. 712, 596-603.

NG, C.-Y. \& Romani, R. W. 2007 Birth kick distributions and the spin-kick correlation of young pulsars. Astrophys. J. 660, 1357-1374.

NG, C.-Y. \& Romani, R. W. 2008 Fitting pulsar wind tori. II. Error analysis and applications. Astrophys. J. 673, 411-417.

Ng, C.-Y., Romani, R. W., Brisken, W. F., Chatterjee, S. \& Kramer, M. 2007 The origin and motion of PSR J0538+2817 in S147. Astrophys. J. 654, 487-493.

PacholczyK, A. G. 1970 Radio Astrophysics. Nonthermal Processes in Galactic and Extragalactic Sources. W. H. Freeman \& Co.

Pavan, L., Bordas, P., Pühlhofer, G., Filipović, M. D., De Horta, A., O’Brien, A., Balbo, M., Walter, R., Bozzo, E., Ferrigno, C. et al. 2014 The long helical jet of the lighthouse nebula, IGR J11014-6103. Astron. Astrophys. 562, A122.

Pavan, L., Pühlhofer, G., Bordas, P., Audard, M., Balbo, M., Bozzo, E., Eckert, D., Ferrigno, C., Filipović, M. D., Verdugo, M. et al. 2016 Closer view of the IGR J11014-6103 outflows. Astron. Astrophys. 591, A91.

Pavlov, G. G., Bhattacharyya, S. \& Zavlin, V. E. 2010 New X-ray observations of the Geminga pulsar wind nebula. Astrophys. J. 715, 66-77.

Petre, R., Kuntz, K. D. \& Shelton, R. L. 2002 The X-ray structure and spectrum of the pulsar wind nebula surrounding PSR B1853+01 in W44. Astrophys. J. 579, 404-410.

Pierbattista, M., Harding, A. K., Gonthier, P. L. \& Grenier, I. A. 2016 Young and middle age pulsar light-curve morphology: comparison of Fermi observations with $\gamma$-ray and radio emission geometries. Astron. Astrophys. 588, A137.

Plucinsky, P. P., Dickel, J. R., Slane, P. O., Edgar, R. J., Gaetz, T. J. \& Smith, R. K. 2002 Chandra observations of the synchrotron nebula in MSH15-56. In APS Meeting Abstracts, American Physical Society.

Porth, O., Vorster, M. J., Lyutikov, M. \& Engelbrecht, N. E. 2016 Diffusion in pulsar wind nebulae: an investigation using magnetohydrodynamic and particle transport models. Mon. Not. R. Astron. Soc. 460, 4135-4149.

Posselt, B., Pavlov, G. G., Slane, P. O., Romani, R., Bucciantini, N., Bykov, A. M., Kargaltsev, O., Weisskopf, M. C. \& NG, C.-Y. 2017 Geminga's puzzling pulsar wind nebula. Astrophys. J. 835, 66.

Rangelov, B., Pavlov, G. G., Kargaltsev, O., Durant, M., Bykov, A. M. \& KRASSILCHTCHIKOV, A. 2016 First detection of a pulsar bow shock nebula in Far-UV: PSR J0437-4715. Astrophys. J. 831, 129. 
Rangelov, B., Pavlov, G. G., Kargaltsev, O., Reisenegger, A., Guillot, S., van KerKWIJK, M. H. \& REYES, C. 2017 Hubble space telescope detection of the millisecond pulsar j2124-3358 and its far-ultraviolet bow shock nebula. Astrophys. J. 835 (2), 264.

Reardon, D. J., Hobbs, G., Coles, W., Levin, Y., Keith, M. J., Bailes, M., Bhat, N. D. R., Burke-Spolaor, S., DAI, S., KerR, M. et al. 2016 Timing analysis for 20 millisecond pulsars in the parkes pulsar timing array. Mon. Not. R. Astron. Soc. 455, 1751-1769.

Reynolds, S. P., Pavlov, G. G., Kargaltsev, O., Klingler, N., Renaud, M. \& Mereghetti, S. 2017 Pulsar-wind nebulae and magnetar outflows: observations at radio, X-ray, and gammaray wavelengths. Space Sci. Rev. 207, 175-234.

Romani, R. W., NG, C.-Y., Dodson, R. \& BRisken, W. 2005 The complex wind torus and jets of PSR B1706-44. Astrophys. J. 631, 480-487.

Romani, R. W., Shaw, M. S., Camilo, F., Cotter, G. \& Sivakoff, G. R. 2010 The balmerdominated bow shock and wind nebula structure of $\gamma$-ray pulsar PSR J1741-2054. Astrophys. $J$. 724, 908-914.

Romanova, M. M., Chulsky, G. A. \& Lovelace, R. V. E. 2005 Winds, B-Fields, and magnetotails of pulsars. Astrophys. J. 630, 1020-1028.

Slane, P., Gaensler, B. M., Van Der Swaluw, E., Hughes, J. P. \& Jenkins, J. A. 2004 Chandra observations of G327.1-1.1: evidence for a disrupted PWN. In American Astronomical Society Meeting Abstracts, Bulletin of the American Astronomical Society, vol. 36, p. 1481. American Astronomical Society.

Stappers, B. W., Gaensler, B. M., Kaspi, V. M., van Der Klis, M. \& Lewin, W. H. G. 2003 An X-ray nebula associated with the millisecond pulsar B1957+20. Science 299, 1372-1374.

Swartz, D. A., Pavlov, G. G., Clarke, T., Castelletti, G., Zavlin, V. E., Bucciantini, N., Karovska, M., van der Horst, A. J., Yukita, M. \& Weisskopf, M. C. 2015 High spatial resolution X-ray spectroscopy of the IC 443 pulsar wind nebula and environs. Astrophys. J. 808, 84.

Temim, T., Slane, P., Castro, D., Plucinsky, P. P., Gelfand, J. \& Dickel, J. R. 2013 High-energy emission from the composite supernova remnant MSH 15-56. Astrophys. J. 768, 61.

Tomsick, J. A., Bodaghee, A., Rodriguez, J., Chaty, S., Camilo, F., Fornasini, F. \& RAHOUI, F. 2012 Is IGR J11014-6103 a pulsar with the highest known kick velocity? Astrophys. J. Lett. 750, L39.

VAN KERKWIJK, M. H. \& INGLE, A. 2008 Reconstructing the guitar: blowing bubbles with a pulsar bow shock backflow. Astrophys. J. Lett. 683, L159.

VAN KerkWIJK, M. H. \& KUlKarni, S. R. 2001 An unusual $H \alpha$ nebula around the nearby neutron star RX J1856.5-3754. Astron. Astrophys. 380, 221-237.

Vigelius, M., Melatos, A., Chatterjee, S., Gaensler, B. M. \& Ghavamian, P. 2007 Threedimensional hydrodynamic simulations of asymmetric pulsar wind bow shocks. Mon. Not. R. Astron. Soc. 374, 793-808.

Voisin, F., Rowell, G., Burton, M. G., Walsh, A., Fukui, Y. \& Aharonian, F. 2016 ism gas studies towards the TeV PWN HESS J1825-137 and northern region. Mon. Not. R. Astron. Soc. 458, 2813-2835.

Wang, Q. D., GotThelf, E. V., ChU, Y.-H. \& Dickel, J. R. 2001 Detection of an X-ray pulsar wind nebula and tail in SNR N157B. Astrophys. J. 559, 275-281.

Wang, Q. D., LI, Z.-Y. \& Begelman, M. C. 1993 The X-ray-emitting trail of the nearby pulsar PSR1929+10. Nature 364, 127-129.

Yao, J. M., Manchester, R. N. \& WANG, N. 2017 A new electron-density model for estimation of pulsar and FRB distances. Astrophys. J. 835, 29.

Yusef-ZAdeh, F. \& GAensler, B. M. 2005 A radio study of the mouse, G359.23-0.82. Adv. Space Res. 35, 1129-1136.

Zeiger, B. R., Brisken, W. F., Chatterjee, S. \& Goss, W. M. 2008 Proper motions of PSRs B1757-24 and B1951+32: implications for ages and associations. Astrophys. J. 674, 271-277. 